\title{
Collective Electron Dynamics in Metallic and Semiconductor Nanostructures
}

\author{
G. Manfredi ${ }^{1}$, P.-A. Hervieux ${ }^{1}$, Y. Yin ${ }^{1}$, and N. Crouseilles ${ }^{2}$ \\ 1 Institut de Physique et Chimie des Matériaux de Strasbourg, 23 Rue du Loess, \\ BP 43, F-67034 Strasbourg, France giovanni.manfredi@ipcms.u-strasbg.fr \\ 2 Institut de Recherche en Mathématiques Avancées, Université Louis Pasteur, \\ Strasbourg, France crouseil@math.u-strasbg.fr
}

\section{Introduction}

Understanding the electron dynamics and transport in metallic and semiconductor nanostructures - such as metallic nanoparticles, thin films, quantum wells and quantum dots - represents a considerable challenge for today's condensed matter physics, both fundamental and applied.

Experimentally, thanks to the recent development of ultrafast spectroscopy techniques, it is now possible to monitor the femtosecond dynamics of an electron gas confined in metallic nanostructures such as thin films $[1,2,3,4$, $5,6,7,8]$, nanotubes [9], metal clusters [10, 11] and nanoparticles $[6,7,12,13]$. Therefore, meaningful comparisons between experimental measurements and numerical simulations based on microscopic theories are becoming possible.

The dynamics of an electron gas confined in a metallic nanostructure is characterised by the presence of collective oscillations (surface plasmon) whose spectral properties depend on several conditions of temperature, density, and coupling to the environment. At lowest order, the linear response of the electron gas is simply given by the plasma frequency $\omega_{p}=\left(e^{2} n / m \varepsilon_{0}\right)^{1 / 2}$ (up to a dimensionless geometrical factor), and does not depend on the temperature or the size of the nano-object. The plasma frequency represents the typical oscillation frequency for electrons immersed in a neutralizing background of positive ions, which is supposed to be motionless because of the large ion mass. The oscillations arise from the fact that, when some electrons are displaced (thus creating a net positive charge), the resulting Coulomb force tends to pull back the electrons towards the excess positive charge. Due to their inertia, the electrons will not simply replenish the positive region, but travel further away thus re-creating an excess positive charge. This effect gives rise to coherent oscillations at the plasma frequency. Notice that, for a metallic nanostructure, the inverse plasma frequency is typically of the order of the femtosecond - this coherent regime can therefore be explored with the ultrafast spectroscopy techniques developed in the last two decades. 
The coherence of such collective motions is progressively destroyed by Landau damping (i.e. by coupling to the internal degrees of freedom of the electron gas) and by electron-electron or electron-phonon collisions. The damping of the plasmon was observed experimentally in gold nanoparticles [14] and was studied theoretically in several works $[15,16,17]$.

Although the linear response of the surface plasmon has been known for a long time, fully nonlinear studies have only been performed in the last decade and have revealed some interesting features. Our own contribution to this research area has mainly focussed on the nonlinear electron dynamics in thin metal films, where the emergence of ballistic low-frequency oscillations has been pointed out [18].

On the other hand, the same type of collective electron motion is also observed in semiconductor nanostructures, such as quantum wells and quantum dots. Although the spatial and temporal scales differ by several order of magnitudes with respect to metallic nanostructures (due the large difference in the electron density), the relevant dimensionless parameters take similar values in both cases [19]. For instance, the effective Wigner-Seitz radius is of order unity for both metallic and semiconductor nano-objects. Therefore, the electron dynamics can be investigated using similar models and both types of nano-objects are expected to share a number of similar dynamical properties.

In this review article, we will describe the collective electron dynamics in metallic and semiconductor nanostructures using different, but complementary, approaches. For small excitations (linear regime), the spectral properties can be investigated via quantum mean-field models of the TDLDA type (timedependent local density approximation), generalized to account for a finite electron temperature. In order to explore the nonlinear regime (strong excitations), we will adopt a phase-space approach that relies on the resolution of kinetic equations in the classical phase space (Vlasov and Wigner equations). The phase-space approach provides a useful link between the classical and quantum dynamics and is well suited to model effects beyond the mean field approximation (electron-electron and electron-phonon collisions). We will also develop a quantum hydrodynamic model, based on velocity moments of the corresponding Wigner distribution function: this approach should lead to considerable gains in computing time in comparison with simulations based on conventional methods, such as density functional theory (DFT).

The above studies all refer to the charge dynamics in a semiconductor or metallic nanostructure, which has been intensively studied in the last three decades. In more recent years, there has been a surge of interest in the spin dynamics of the carriers, mainly for possible applications to the emerging field of quantum computing [20]. A promising approach to the development of a quantum computer relies on small semiconductor devices, such as quantum dots and quantum wells [21]. To implement basic qubit operations, most proposed schemes make use of the electron spin states, so that a thorough understanding of the spin dynamics is a necessary prerequisite. Nevertheless, in order to manipulate the electrons themselves, one must necessarily resort to 
electromagnetic fields, which in turn excite the Coulomb mean field [22, 23]. The charge and spin dynamics are therefore closely intertwined and both must be taken into account for a realistic modelling of semiconductor-based qubit operations.

The ultrafast magnetization (spin) dynamics in ferromagnetic nanostructures has also attracted considerable experimental attention in the last decade. Pioneering experiments [24] on ferromagnetic thin films revealed that the magnetization experiences a rapid drop (on a femtosecond time scale) when the films is irradiated with an ultrafast laser pulse, after which it slowly regains its original value on a time scale close to that of the electron-phonon coupling. Despite many attempts $[24,25,26]$, a clear theoretical explanation for these effects is still lacking. Here, we will illustrate how this problem can be addressed using some of the techniques developed for the electron dynamics, particularly quantum mean-field and phase-space methods, which will be generalized to include the spin degrees of freedom.

\section{Models for the electron dynamics}

Metallic and semiconductor nano-objects operate in very different regimes, as the electron density is several orders of magnitudes larger for the former. Consequently, the typical time, space, and energy scales can be very different, as illustrated in Table 1. However, if one takes into account the effective electron mass and dielectric constant, the relevant dimensionless parameters turn out to be rather similar [19]: for instance, from Table 1 we see immediately that the ratio of the screening length $\left(L_{\text {screen }}=v_{F} / \omega_{p}\right.$, where $v_{F}$ is the Fermi velocity) to the effective Bohr radius $a_{B}=4 \pi \varepsilon \hbar^{2} / m e^{2}$ is of order unity. The same happens for the ratio of the plasmon energy $\hbar \omega_{p}$ to the Fermi energy $E_{F}$, so that the normalized Wigner-Seitz radius $r_{s}$ is also of order unity for both cases. ${ }^{3}$

It is therefore not surprising that the electron dynamics of both types of nanostructures can be described by means of similar models. A bird's-eye view of the various relevant models is provided in Fig. 1. The diagram represents the various levels of modeling for the electron dynamics, both quantum (left column, orange) and classical (right column, blue). The highest level of description is the $N$-body model, which involves the resolution of the $N$-particle Schrödinger equation in the quantum regime, or the $N$-particle Liouville equation for classical problems (the latter is of course equivalent to Newton's equations of motion). This is a difficult task even classically, although molecular dynamics simulations that solve the exact $N$-body problem can nowadays attain

\footnotetext{
${ }^{3}$ For a quantum well, all relevant lengths far exceed the semiconductor lattice spacing $a_{\text {latt }} \simeq 5 \AA$. This makes semiconductor systems a much better approximation to jellium (i.e., a continuum ionic density profile) than simple metals, for which the lattice spacing is comparable to the other electronic lengths.
} 
Table 1. Typical time, space, and energy scales for metallic and semiconductor nanostructures

\begin{tabular}{lll}
\hline & \multicolumn{2}{l}{ Metal film Quantum well } \\
\hline$n_{e}$ & $10^{28} \mathrm{~m}^{-3}$ & $10^{22} \mathrm{~m}^{-3}$ \\
$m$ & $m_{e}$ & $m_{*} \simeq 0.07 m_{e}$ \\
$\varepsilon$ & $\varepsilon_{0}$ & $\varepsilon \simeq 12 \varepsilon_{0}$ \\
$L_{\text {screen }}$ & $1 \AA$ & $100 \AA$ \\
$\omega_{p}^{-1}$ & $1 \mathrm{fs}$ & $1 \mathrm{ps}$ \\
$E_{F}$ & $1 \mathrm{eV}$ & $1 \mathrm{meV}$ \\
$T_{F}$ & $10^{4} \mathrm{~K}$ & $10 \mathrm{~K}$ \\
$a_{B}$ & $0.529 \AA$ & $100 \AA$ \\
$a_{\text {latt }}$ & $5 \AA$ & $5 \AA$ \\
$r_{s} / a_{B}$ & 5 & 3 \\
\hline
\end{tabular}

a considerable level of sophistication. For Newton's equations with two-body interactions, the numerical complexity grows at most as $N^{2}$, and in some cases this can be reduced to a logarithmic dependence. Quantum-mechanically, the $N$-body problem is virtually unmanageable, except for very small systems, because the size of the relevant Hilbert space grows exponentially with $N$. Nevertheless, exact simulations of the $N$-body Schrödinger equation can be performed using the so-called configuration interaction (CI) method. We have used this approach to study the exact electron dynamics in semiconductor quantum dots containing up to four electrons.

For larger systems, some rather drastic approximations need to be made if we want to end up with a mathematically and numerically tractable model. Most such reduced models are improvements on the so-called 'mean field approximation', which states that the motion of a single electron is determined by the positions and velocities of all other particles in the system. Such collective behavior is possible because of the long-range nature of electromagnetic forces. The mean field approach can be viewed as a zeroth-order approximation to the $N$-body problem in which two-body (and higher order) correlations between the particles have been neglected. Classically, this procedure is known as the BBGKY hierarchy (from the names of Bogoliubov, Born, Green, Kirkwood, and Yvon) [27].

For classical systems of charged particles (plasmas), the mean-field dynamics is governed by the Vlasov equation, which describes the evolution of a one-particle probability density in the phase space. The quantum analog of the Vlasov equation is provided by the time-dependent Hartree equations, which are actually one-body Schrödinger equations evolving in the mean-field potential. In both cases, the mean field is obtained by solving Maxwell's equations, often reduced, in the electrostatic limit, to the sole Poisson's equation.

In this review, we concentrate on quantum mechanical models. Several improvements have been proposed to the Hartree equations (which were derived 
in 1927, just one year after Schrödinger's seminal paper on the wave equation), most notably Fock's correction (1930). Indeed, the Hartree method does not respect the principle of antisymmetry of the wavefunction, although it does use the Pauli exclusion principle in its less stringent formulation, forbidding the presence of two electrons in the same quantum state. The Hartree-Fock equations respect the antisymmetry of the wavefunctions, thus leading to an extra interaction term between the electrons, termed the "exchange interaction'.

A particularly successful extension of the mean-field approach is the density-functional theory (DFT), which was developed by Hohenberg, Kohn, and Sham in the mid 1960s [28]. Originally developed for the ground state at zero temperature, it has subsequently been extended to finite temperature and time-dependent problems. As its name suggests, DFT states that all the properties of a many-electron systems are determined by the electron spatial density, rather than by the wavefunctions. DFT allows one to introduce in the mean-field formalism effects that go beyond the strict mean-field approximation, particularly the exchange interaction described above. Indeed, DFT can deal with higher order correlations between the electrons, in principle exactly if the exact density functional were known. In practice, one has to make an educated guess for the appropriate correlation functional, which leads to various empirical approximations. Nevertheless, DFT has proven immensely useful for a wide range of electronic structure calculations.

The Hartree equations can be equivalently recast in a phase-space formalism by making use of the Wigner transformation, which was introduced by E. Wigner in 1932 [29]. The resulting Wigner function is a pseudo probability distribution, which can be used to compute expectation values just like its classical counterpart. Unfortunately, the Wigner function can take negative values, which precludes the possibility of interpreting it as a true probability density.

By taking velocity moments of the Wigner equation - and using some appropriate closure hypotheses - one can derive a set of quantum hydrodynamical (or fluid) equations that govern the evolution of macroscopic quantities such as the particle density, average velocity, pressure, heat flux etc. Compared to the Wigner approach, the hydrodynamical one is obviously numerically advantageous, as it requires the resolution of a small number of equations in real (not phase) space. Generally speaking, hydrodynamical methods yield accurate results over distances that are larger than the typical electrostatic screening length, which is the Debye length $\lambda_{D}=\left(k_{B} T_{e} \varepsilon / e^{2} n\right)^{1 / 2}$ for classical plasmas and the Thomas-Fermi screening length $L_{F}=v_{F} / \omega_{p}$ for degenerate electron gases (see Table 1).

In the following subsections, we shall present a brief overview of most of the quantum models featuring in Fig. 1. 


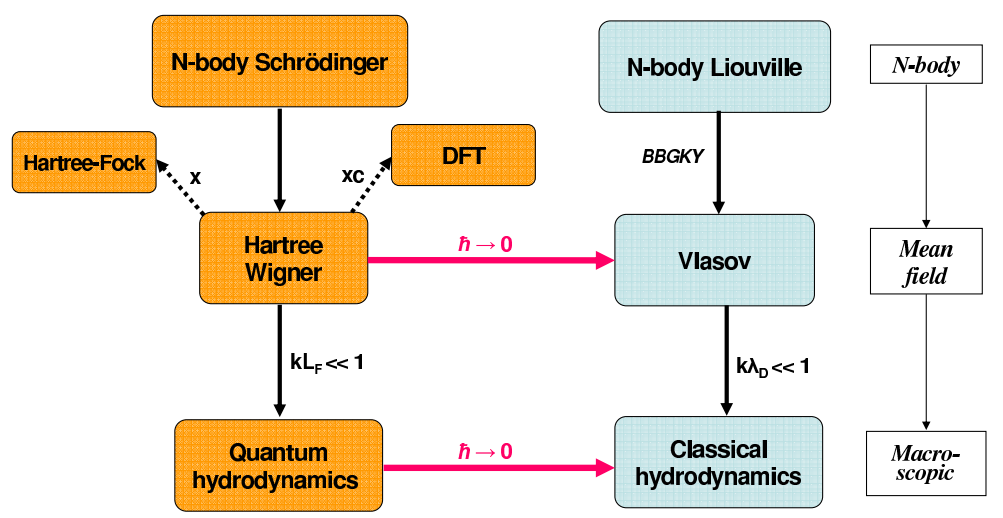

Fig. 1. Bird's-eye view of the models used to describe the electron dynamics. From top to bottom: $N$-body, mean-field, and macroscopic (hydrodynamic) theory. Left column (orange): quantum models; right column (blue): classical models. Notation: $\mathrm{x}$ $=$ exchange $; \mathrm{xc}=$ exchange and correlations; $\lambda_{D}=$ Debye length (classical screening length); $L_{F}=$ Thomas-Fermi screening length; $\mathrm{k}=$ typical wavevector; BBGKY = Bogoliubov, Born, Green, Kirkwood, Yvon hierarchy. 
2.1 Exact $N$-body simulations: the Configuration Interaction (CI) method

\section{Method}

In the Hartree-Fock model (HF), the many-body wave-function is approximated by a single Slater determinant leading to a correlation between electrons having the same spin. However, electrons of different spin are not correlated in this approximation. This is why the difference between the exact value of the energy, and the HF value is called the correlation energy. There are a number of quantum chemistry methods, which attempt to improve the description of the many-body wave-function. The most important one is the so-called configuration interaction method (CI) [30] which is based on the minimization of the energy with respect to the expansion coefficients of a trial many-body wave-function expressed as a linear combination of Slater determinants. With respect to the models based on density functional methods the drawback of the CI method is its unfavorable scaling with the system size. Indeed, the dimension of a full CI calculation grows factorially with the number of electrons and basis functions.

From the above considerations, it is clear that CI calculations are restricted to confined systems with very few electrons (typically less than 10). In quantum chemistry, the "basis set" usually refers to the set of (nonorthogonal) one-particle functions used to build molecular orbitals. Concerning the computational methodology for confined electron systems (atoms, molecules, clusters, nanoparticles, quantum dots...) localized basis sets are the traditional choice and the most common type of basis functions is the Gaussian functions. It is worth noticing that, from the knowledge of the exact many-body wave-function, one can in principle: (i) compute the temporal evolution of the system, including the dynamical correlations; (ii) obtain the true excited states of the system.

In the following, an application of the CI method in the field of semiconductor nanostructures and quantum dots is presented.

\section{Application}

Recent progress in semiconductors technology allows the realization of quantum systems composed of a small number of electrons (even a single electron!) confined in nanometer-scale potential wells. These systems, which provide highly tunable structures for trapping and manipulating individual electrons, are often named artificial atoms or quantum dots and are good candidates for the emerging technology of quantum computing. They have certain similarities with atoms in the sense that they have a discrete electronic structure that follows the well-known Hund's rule of atomic physics. However, in quantum dots the electrons are generally confined by harmonic or quasi-harmonic potentials, whereas atoms are characterized by Coulomb confinement potentials. The spectral properties of quantum dots are exotic with respect to the 
properties of atoms in the sense that most of the oscillator strength is concentrated almost exclusively on one dipolar transition. This property is a direct consequence of Kohn's theorem (KT) and does not depend on the number of electrons, the strength of the confinement or the electron-electron interaction [31].

In a recent work [32], we have investigated quasi-two-dimensional Gaussian quantum dots containing up to four electrons within the framework of the CI method which allows in principle an exact treatment of the many-electron system. The Schrödinger equation for $N$-electrons confined by a potential $V_{e x t}$ is given by

$$
H \Psi(1, \ldots, N)=E \Psi(1, \ldots, N)
$$

where $(1, \ldots, N)$ represents the space $\left[\boldsymbol{r}_{i}=\left(x_{i}, y_{i}, z_{i}\right)\right]$ and spin coordinates of the electrons and

$$
H=\sum_{i=1}^{N}-\frac{\hbar^{2}}{2 m} \nabla_{i}^{2}+\sum_{i>j}^{N} \frac{e^{2}}{4 \pi \epsilon\left|\boldsymbol{r}_{i}-\boldsymbol{r}_{j}\right|}+\sum_{i=1}^{N} V_{e x t}\left(\boldsymbol{r}_{i}\right) .
$$

The confinement is modelled by an external one-particle anisotropic Gaussian potential given by

$$
V_{\text {ext }}\left(\boldsymbol{r}_{i}\right)=-D \exp \left[-\gamma\left(x_{i}^{2}+y_{i}^{2}\right)\right]+\frac{1}{2} m^{2} \omega_{z}^{2} z_{i}^{2} .
$$

It is worth noticing that for sufficiently large values of $\omega_{z}$ the electrons of the system are strongly compressed along the $z$ direction. Therefore, in this situation, the system can be regarded as a quantum system confined by a twodimensional Gaussian-type potential, i.e., as a quasi-two-dimensional Gaussian quantum dot. Since a Gaussian potential can be approximated close to its minimum by an harmonic potential, the potential of Eq. (3) is suitable for the modelling of anharmonic quantum dots. The anharmonicity of the confinement can be characterized by the depth of the Gaussian potential $D$ and by the quantity $\omega=\sqrt{2 D \gamma} / \mathrm{m}$. Thus, when $D$ is much larger than $\hbar \omega$ the Gaussian potential has many bound states and the potential curve follows closely the harmonic oscillator potential leading to a small anharmonicity of the system. On the other hand, when $D$ is slightly larger than $\hbar \omega$ the Gaussian potential has only few bound states and, therefore, deviates strongly from the harmonic potential leading to a large anharmonicity. Also, a large (small) value of $\omega$ corresponds to a strong (weak) confinement with respect to the electron-electron interaction.

The wave-function is approximated by a linear combination of cartesian anisotropic Gaussian-Type Orbitals (c-aniGTO) [33]. A c-aniGTO centered at $\left(b_{x}, b_{y}, b_{z}\right)$ is defined as

$$
\chi^{\boldsymbol{a}, \boldsymbol{\zeta}}(\boldsymbol{r}, \boldsymbol{b})=x_{b_{x}}^{a_{x}} y_{b_{y}}^{a_{y}} z_{b_{z}}^{a_{z}} \exp \left(-\zeta_{x} x_{b_{x}}^{2}-\zeta_{y} y_{b_{y}}^{2}-\zeta_{z} z_{b_{z}}^{2}\right)
$$

where $x_{b_{x}}=\left(x-b_{x}\right)$ etc...Following the quantum chemical convention the orbitals are classified as $s$-type, $p$-type, for $l=a_{x}+a_{y}+a_{z}=0,1, \ldots$, respectively (this sum controls the value of the orbital angular momentum). The 
$\left(b_{x}, b_{y}, b_{z}\right)$ parameters have been chosen to coincide with the center of the confining potential. This type of basis sets was found to be the most suitable one for expanding the eigenfunctions of an electron in an anisotropic harmonic oscillator potential. The calculations have been performed using the OpenMol Program ${ }^{4}$.

Energy spectra and oscillator strengths have been calculated for different strength of confinement $\omega$ and potential depth $D$. The effect of the electronelectron interaction on the distribution of oscillator strengths and the breakdown of the KT has been examined by focusing on the results with the same value of $D / \hbar \omega$ i.e. with the same anharmocity.

A substantial red-shift has been observed for the dipole transitions corresponding to the excitation into the center-of-mass mode. The oscillator strengths, which are concentrated exclusively in the center-of-mass excitation in the harmonic limit, are distributed among the near-lying transitions as a result of the breakdown of the Kohn's theorem. The distribution of the oscillator strengths is limited to the transitions located in the lower-energy region when $\omega$ is large (i.e. for strongly confined electrons) but it extends towards the higher-energy region when $\omega$ becomes small (i.e. for weakly confined electrons).

The analysis of the CI wave functions shows that all states can be classified according to the polyad quantum number $v_{p}$ [32]. The distribution of the oscillator strengths for large $\omega$ occurs among transitions involving excited states with the same value of $v_{p}$ as the center-of-mass excited state, $v_{p, c m}$, while it occurs among transitions involving the excited states with $v_{p}=v_{p, c m}$ and $v_{p}=v_{p, c m+2}$ for small $\omega$.

\subsection{Time-Dependent Density Functional Theory (TDDFT) and the Local-Density Approximation (LDA)}

Time dependent density functional theory (TDDFT) extends the basic ideas of static density functional theory (DFT) to the more general situation of systems under the influence of time dependent external fields. This dynamical approach relies on the electron density $n(\boldsymbol{r}, t)$ rather than on the many-body wave function $\Psi\left(\boldsymbol{r}_{1}, \boldsymbol{r}_{2}, \ldots, \boldsymbol{r}_{N}, t\right)$ of the system. In fact, the central theorem of the TDDFT is the Runge-Gross theorem [34, 35, 36] which tells us that all observables are uniquely determined by the density.

From the computational point of view, with respect to the resolution of the time dependent Schrödinger equation (TDSE) of an $N$-electron system, the complexity is strongly reduced when using TDDFT. Indeed, the wave function depends on $3 N+1$ variables $\left(\boldsymbol{r}_{1}, \boldsymbol{r}_{2}, \ldots, \boldsymbol{r}_{N}, t\right)$ while the density depends only on 4 variables $(\boldsymbol{r}, t)$. This is one of the reasons why this method has become so popular. A practical scheme for computing $n(\boldsymbol{r}, t)$ is provided by

\footnotetext{
${ }^{4}$ see http://www.csc.fi/gopenmol
} 
the Kohn-Sham (KS) formulation of the TDDFT [28]. In the latter, noninteracting electrons are moving in an effective local potential constructed in such a way that the KS density is the same as the one of the interacting electron system. The advantage of this formulation lies in its computational simplicity compared to other quantum-chemical methods such as time-dependent Hartree-Fock or configuration interaction. The KS equations read as

$$
i \hbar \frac{\partial}{\partial t} \phi_{k}(\boldsymbol{r}, t)=\left(-\frac{\hbar^{2}}{2 m} \nabla^{2}+V_{e f f}(\boldsymbol{r}, t)\right) \phi_{k}(\boldsymbol{r}, t)
$$

with the KS density

$$
n(\boldsymbol{r}, t)=\sum_{k=1}^{\infty} f_{k}\left|\phi_{k}(\boldsymbol{r}, t)\right|^{2}
$$

where $f_{k}$ denotes the occupation numbers of the ground state, and

$$
V_{e f f}(\boldsymbol{r}, t)=V_{e x t}(\boldsymbol{r}, t)+V_{H}(\boldsymbol{r}, t)+V_{x c}(\boldsymbol{r}, t) .
$$

In the above expression the first term is the external potential (ionic potential, laser field...), the second is the Hartree potential, which is a solution of the Poisson's equation, and the last term is the exchange-correlation potential.

The most popular choice for $V_{x c}$ is the so-called adiabatic local density approximation (ALDA) given by

$$
V_{x c}(\boldsymbol{r}, t)=\frac{d}{d n}\left[n \epsilon_{x c}(n)\right]_{n=n(\boldsymbol{r}, t)},
$$

where $\epsilon_{x c}(n)$ is the exchange-correlation energy density for an homogeneous electron gas of density $n$. In this approach, the same functional used to calculate the properties of the ground state is employed in the dynamical simulations.

The validity of the local approximation has been discussed in many papers and textbooks [37]. This approximation works remarkably well for inhomogeneous electron systems. In contrast, the validity of the adiabatic approximation has been less thoroughly analyzed. Generally speaking, this approach is expected to hold for finite systems and for processes that evolve very slowly in time. The situation in bulk solids is more controversial since significant deficiencies in the description of absorption spectra have been noticed [38]. It was shown by Dobson [39] that ALDA fulfills the Kohn theorem when applied to a system of interacting electrons confined in an external parabolic potential. This theorem guarantees the existence of a collective state at the same frequency as the harmonic potential. It corresponds to a rigid oscillation of the many-body wavefunction around the center of the external potential.

Only a few attempts have been made to go beyond ALDA. To date, the most important ones are the work of Gross and Kohn [40] and that of Vignale and Kohn [41], the latter being the most promising in particular for studying electron relaxation phenomena [42]. Contrarily to ALDA, the approach of 
Gross and Kohn, which uses a frequency-dependent parametrization of the exchange-correlation kernel (see below), does not fulfill the Kohn theorem $[31,39]$. This problem was further investigated by Vignale and Kohn [41], who proposed a new theory based on the so-called current density functional theory (CDFT). This model is described in detail in [43]. CDFT was originally derived by Vignale and Rasolt [44] to describe, within the framework of DFT, situations where strong magnetic fields and orbital currents cannot be ignored.

Few works have been devoted to the study of the nonlinear electron dynamics in finite metallic systems exposed to strong external fields. Indeed, the resolution of the time-dependent Kohn-Sham equations (5) is a very difficult task particularly for 3D systems. Some pioneering work on free simple-metal clusters was performed by E. Suraud in Toulouse and P.-G. Reinhard in Erlangen [45]. More recently, Gervais et al. [46] have investigated the same problem in 3D geometry using a spherical basis expansion technique. This approach is restricted to small metal clusters. The interaction of strong femtosecond laser pulses with a $\mathrm{C}_{60}$ molecule (which possesses 240 delocalized electrons and can therefore be considered as a metallic nano-object [47]) has been investigated in Ref. [48] by employing a TDDFT approach. Still concerning the fullerene molecule, Cormier et al. [49] studied multiphoton absorption processes by solving numerically the associated time-dependent Schrödinger equation (TDSE) in the single active electron (SAE) approximation. This approximation consists in solving the equations (5) by using, instead of the time-dependent effective potential $V_{\text {eff }}(\boldsymbol{r}, t)$ given in Eq. (7), the static effective potential of the ground state together with the time-dependent electric potential of the laser.

Let us now examine the linear regime, which has received much wider attention in the past.

Under the condition that the external field is weak, the simplest way to implement TDDFT is to work within the framework of the linear response theory. This approximation was first introduced by Zangwill and Soven [50] in the context of atomic physics for the study of photoionization in rare gases. Subsequently, this formalism has been successfully extended to the study of more and more complex electron systems: molecules [51], simple metal clusters [52], noble metal clusters [53], thin metal films [54], quantum dots [55], and condensed phase systems [38].

To date, in the field of nanoparticle physics, most applications of the timedependent Kohn-Sham formalism have been performed at zero electron temperature. In order to interpret time-resolved pump-probe experiments carried out on noble metal nanoparticles, we have recently extended this approach to finite temperature. In the following we provide a brief overview of the model with the basic equations. 


\section{Ground state}

The electron gas is assumed to be at thermal equilibrium with temperature $T_{e}$. In the Kohn-Sham formulation of the density functional theory at finite temperature within the grand-canonical ensemble [56], the ground-state electron density $n$ of an $N$-electron system is written, in terms of single-particle orbitals $\phi_{i}$ and energies $\varepsilon_{i}$, as

$$
n(\boldsymbol{r})=\sum_{k=1}^{\infty} f_{k} n_{k}(\boldsymbol{r})=\sum_{k=1}^{\infty} f_{k}\left|\phi_{k}(\boldsymbol{r})\right|^{2}
$$

where $f_{k}=\left[1+\exp \left\{\left(\varepsilon_{k}-\mu\right) / k_{B} T_{e}\right\}\right]^{-1}$ are the Fermi occupation numbers and $\mu$ is the chemical potential. These orbitals and energies obey the Schrödinger equation

$$
\left[-\frac{\hbar^{2}}{2 m} \nabla^{2}+V_{e f f}(\boldsymbol{r})\right] \phi_{i}(\boldsymbol{r})=\varepsilon_{i} \phi_{i}(\boldsymbol{r}),
$$

where $V_{\text {eff }}(\boldsymbol{r})$ is an effective single-particle potential given by

$$
V_{e f f}(\boldsymbol{r})=V_{e x t}(\boldsymbol{r})+V_{H}(\boldsymbol{r})+V_{x c}(\boldsymbol{r}),
$$

where $V_{\text {ext }}(\boldsymbol{r})$ is an external potential (e.g. due to the ionic background), $V_{H}(\boldsymbol{r})$ is the Hartree potential solution of Poisson's equation, and $V_{x c}(\boldsymbol{r})$ is the exchange-correlation potential defined by

$$
V_{x c}(\boldsymbol{r})=\frac{d}{d n}\left[n \omega_{x c}(n)\right]_{n=n(\boldsymbol{r})},
$$

where $\Omega_{x c}(n) \equiv \int n(\boldsymbol{r}) \omega_{x c}(n(\boldsymbol{r})) d \boldsymbol{r}$ is the exchange-correlation thermodynamic potential [57]. The temperature appears in the self-consistent procedure only through the occupation numbers and the exchange-correlation thermodynamic potential.

For low temperature (i.e. $T_{e} \ll T_{F}[n(\boldsymbol{r})]$ where $T_{F}[n(\boldsymbol{r})]=\frac{\hbar^{2}}{2 m k_{B}}\left(3 \pi^{2} n(\boldsymbol{r})\right)^{2 / 3}$ is the local Fermi temperature), $\omega_{x c}(n)$ may be safely replaced by its value at $T_{e}=0$, i.e. by $\epsilon_{x c}(n)$. The chemical potential is determined self-consistently by requiring the conservation of the total number of electrons from Eq. (9) $[58,59]$.

\section{Excited States}

In the usual first-order TDLDA at $T_{e}=0$ in the frequency domain, the induced electron density $\delta n(\boldsymbol{r} ; \omega)$ is related to $\delta V_{e x t}\left(\boldsymbol{r}^{\prime} ; \omega\right)$, the Fourier transform (with respect to time) of the external time-dependent potential (generated, for instance, by the electric field of a laser beam), via the relation $[50,52,60]$ 


$$
\delta n(\boldsymbol{r} ; \omega)=\int \chi\left(\boldsymbol{r}, \boldsymbol{r}^{\prime} ; \omega\right) \delta V_{e x t}\left(\boldsymbol{r}^{\prime} ; \omega\right) d \boldsymbol{r}^{\prime}
$$

where $\chi\left(\boldsymbol{r}, \boldsymbol{r}^{\prime} ; \omega\right)$ is the retarded density correlation function or the dynamic response function. It is possible to rewrite the induced density as

$$
\delta n(\boldsymbol{r} ; \omega)=\int \chi^{0}\left(\boldsymbol{r}, \boldsymbol{r}^{\prime} ; \omega\right) \delta V_{e f f}\left(\boldsymbol{r}^{\prime} ; \omega\right) d \boldsymbol{r}^{\prime}
$$

with

$$
\begin{aligned}
\delta V_{e f f}(\boldsymbol{r} ; \omega) & =\delta V_{e x t}(\boldsymbol{r} ; \omega)+\frac{e^{2}}{4 \pi \epsilon_{0}} \int \frac{\delta n\left(\boldsymbol{r}^{\prime} ; \omega\right)}{\left|\boldsymbol{r}-\boldsymbol{r}^{\prime}\right|} d \boldsymbol{r}^{\prime} \\
& +\int f_{x c}\left(\boldsymbol{r}, \boldsymbol{r}^{\prime} ; \omega\right) \delta n\left(\boldsymbol{r}^{\prime} ; \omega\right) d \boldsymbol{r}^{\prime}
\end{aligned}
$$

where the function $f_{x c}\left(\boldsymbol{r}, \boldsymbol{r}^{\prime} ; \omega\right)$ is the Fourier transform of the time-dependent kernel defined by $f_{x c}\left(\boldsymbol{r}, t ; \boldsymbol{r}^{\prime}, t^{\prime}\right) \equiv \delta V_{x c}(\boldsymbol{r}, t) / \delta n\left(\boldsymbol{r}^{\prime}, t^{\prime}\right)$ and $\chi^{0}\left(\boldsymbol{r}, \boldsymbol{r}^{\prime} ; \omega\right)$ is the non-interacting retarded density correlation function. From Eqs. (13)-(15) we see that $\chi^{0}$ and $\chi$ are related by an integral equation (Dyson-type equation)

$$
\begin{aligned}
\chi\left(\boldsymbol{r}, \boldsymbol{r}^{\prime} ; \omega\right) & =\chi^{0}\left(\boldsymbol{r}, \boldsymbol{r}^{\prime} ; \omega\right)+\iint \chi^{0}\left(\boldsymbol{r}, \boldsymbol{r}^{\prime \prime} ; \omega\right) \\
& \times K\left(\boldsymbol{r}^{\prime \prime}, \boldsymbol{r}^{\prime \prime \prime} ; \omega\right) \chi\left(\boldsymbol{r}^{\prime \prime \prime}, \boldsymbol{r}^{\prime} ; \omega\right) d \boldsymbol{r}^{\prime \prime} d \boldsymbol{r}^{\prime \prime \prime},
\end{aligned}
$$

with the residual interaction defined by

$$
K\left(\boldsymbol{r}, \boldsymbol{r}^{\prime} ; \omega\right)=\frac{e^{2}}{4 \pi \epsilon_{0}\left|\boldsymbol{r}-\boldsymbol{r}^{\prime}\right|}+f_{x c}\left(\boldsymbol{r}, \boldsymbol{r}^{\prime} ; \omega\right) .
$$

In the adiabatic local-density approximation (ALDA) the exchange-correlation kernel is frequency-independent and local, and reduces to [50, 60]

$$
f_{x c}\left(\boldsymbol{r}, \boldsymbol{r}^{\prime}\right)=\frac{d}{d n}\left[V_{x c}(n)\right]_{n=n(\boldsymbol{r})} \delta\left(\boldsymbol{r}-\boldsymbol{r}^{\prime}\right) .
$$

It should be mentioned that the functional, $V_{x c}$ in the above equation is the same as the one used in the calculation of the ground state [see Eq. (12)]. For spin-saturated electronic systems, we have

$$
\begin{aligned}
\chi^{0}\left(\boldsymbol{r}, \boldsymbol{r}^{\prime} ; \omega\right)= & 2 \sum_{j k}\left[f_{j}^{0}-f_{k}^{0}\right] \frac{\phi_{j}^{*}(\boldsymbol{r}) \phi_{k}(\boldsymbol{r}) \phi_{k}^{*}\left(\boldsymbol{r}^{\prime}\right) \phi_{j}\left(\boldsymbol{r}^{\prime}\right)}{\hbar \omega-\left(\varepsilon_{k}-\varepsilon_{j}\right)+i \eta} \\
= & \sum_{k}^{o c c} \phi_{k}^{*}(\boldsymbol{r}) \phi_{k}\left(\boldsymbol{r}^{\prime}\right) G_{+}\left(\boldsymbol{r}, \boldsymbol{r}^{\prime} ; \varepsilon_{k}+\hbar \omega\right)+ \\
& \sum_{k}^{o c c} \phi_{k}(\boldsymbol{r}) \phi_{k}^{*}\left(\boldsymbol{r}^{\prime}\right) G_{+}^{*}\left(\boldsymbol{r}, \boldsymbol{r}^{\prime} ; \varepsilon_{k}-\hbar \omega\right)
\end{aligned}
$$


where $\phi_{k}(\boldsymbol{r})$ and $\varepsilon_{k}$ are the one-electron Kohn-Sham wave functions and energies, respectively. $G_{+}$is the one-particle retarded Green's function and $f_{k}^{0}$ are the Fermi occupation numbers at $T_{e}=0 \mathrm{~K}(0$ or 1$)$. All the above quantities are obtained with the procedure described in the preceding subsection with $f_{k}=f_{k}^{0}$ in Eq. (9). In order to produce numerically tractable results, we have added a small imaginary part to the probe frequency, so that $\omega \rightarrow \omega+i \delta$ with $\eta=\hbar \delta$.

At finite electron temperature, the grand-canonical non-interacting retarded density correlation function reads [61]

$$
\begin{aligned}
\chi^{0}\left(\boldsymbol{r}, \boldsymbol{r}^{\prime} ; \omega ; T_{e}\right) & =\frac{1}{Z_{G}} \sum_{n, N} \exp \left\{-\frac{1}{k_{B} T_{e}}\left[E_{n}(N)-N \mu\right]\right\} \\
& \times \chi_{n, N}^{0}\left(\boldsymbol{r}, \boldsymbol{r}^{\prime} ; \omega ; T_{e}\right)
\end{aligned}
$$

where $Z_{G}$ is the grand-canonical partition function

$$
Z_{G}=\sum_{n, N} \exp \left\{-\frac{1}{k_{B} T_{e}}\left[E_{n}(N)-N \mu\right]\right\}
$$

with $E_{n}(N)$ the energy of the state $|n N\rangle$ having $N$ electrons, $\mu$ the chemical potential and

$$
\begin{aligned}
\chi_{n, N}^{0}\left(\boldsymbol{r}, \boldsymbol{r}^{\prime} ; \omega ; T_{e}\right) & =\sum_{m} \frac{\langle n N|\hat{n}(\boldsymbol{r})| m N\rangle\left\langle m N\left|\hat{n}\left(\boldsymbol{r}^{\prime}\right)\right| n N\right\rangle}{\hbar \omega-\left(E_{m}(N)-E_{n}(N)\right)+i \eta} \\
& -\frac{\left\langle n N\left|\hat{n}\left(\boldsymbol{r}^{\prime}\right)\right| m N\right\rangle\langle m N|\hat{n}(\boldsymbol{r})| n N\rangle}{\hbar \omega+\left(E_{m}(N)-E_{n}(N)\right)+i \eta} .
\end{aligned}
$$

In the above expression $\hat{n}(\boldsymbol{r})$ is the particle density operator defined from the wave field operators by

$$
\hat{n}(\boldsymbol{r})=\hat{\psi}^{+}(\boldsymbol{r}) \hat{\psi}(\boldsymbol{r})
$$

with $\hat{\psi}^{+}(\boldsymbol{r})=\sum_{k} \hat{a}_{k}^{+} \phi_{k}^{*}(\boldsymbol{r})$ and $\hat{\psi}(\boldsymbol{r})=\sum_{k} \hat{a}_{k} \phi_{k}(\boldsymbol{r})$. By using standard field theory techniques it is possible to show that

$$
\begin{aligned}
\chi^{0}\left(\boldsymbol{r}, \boldsymbol{r}^{\prime} ; \omega ; T_{e}\right) & =\sum_{k} f_{k} \phi_{k}^{*}(\boldsymbol{r}) \phi_{k}\left(\boldsymbol{r}^{\prime}\right) G_{+}\left(\boldsymbol{r}, \boldsymbol{r}^{\prime} ; \varepsilon_{k}+\hbar \omega ; T_{e}\right) \\
& +\sum_{k} f_{k} \phi_{k}(\boldsymbol{r}) \phi_{k}^{*}\left(\boldsymbol{r}^{\prime}\right) G_{+}^{*}\left(\boldsymbol{r}, \boldsymbol{r}^{\prime} ; \varepsilon_{k}-\hbar \omega ; T_{e}\right)
\end{aligned}
$$

where $f_{k}=\left[1+\exp \left\{\left(\varepsilon_{k}-\mu\right) / k_{B} T_{e}\right\}\right]^{-1}$. So far, we have assumed that the residual interaction (17) is temperature independent. This assumption is consistent with the use of $\omega_{x c}(n)=\epsilon_{x c}(n)$ in the calculation of the ground-state properties. Therefore, as for $T_{e}=0$, the response function is solution of the Dyson equation (16) with $\chi^{0}$ given by Eq. (24).

The above formalism can be employed to compute the photo-absorption by a metallic nanoparticle of size $R$. If the wavelength $\lambda$ of the incoming light 
is such that $\lambda \gg R$ the dipolar approximation is valid. From the frequencydependent dipole polarizability

$$
\alpha\left(\omega ; T_{e}\right)=\int \delta n\left(\boldsymbol{r} ; \omega ; T_{e}\right) \delta V_{e x t}(\boldsymbol{r} ; \omega) d \boldsymbol{r}
$$

one obtains the dipolar absorption cross-section [62]

$$
\sigma\left(\omega ; T_{e}\right)=\frac{\omega}{\varepsilon_{0} c} \operatorname{Im}\left[\alpha\left(\omega ; T_{e}\right)\right] .
$$

As for the zero-temperature case, the dipolar absorption cross-section fulfils the well known Thomas-Reiche-Kuhn (TRK) sum rule

$$
\int \sigma\left(\omega ; T_{e}\right) d \omega=\frac{2 \pi^{2} N}{c} .
$$

\section{Application to femtosecond spectroscopy}

Ultrafast spectroscopy using femtosecond laser pulses is a well suited technique to study the electronic energy relaxation mechanisms in metallic nanoparticles (see Refs. [6, 12] and references therein). The experiments have been carried out with nanoparticles of noble metals containing several thousand atoms and embedded in a transparent matrix. By using a time resolved pump-probe configuration it is possible to have access to the spectral and temporal dependence of the differential transmission $\frac{\Delta T}{T}(\tau, \omega)$, defined as the normalized difference between the probe pulse with and without the pump pulse. This quantity contains the information on the electron dynamics, which is measured as a function of the pump-probe time delay $\tau$ and of the laser frequency $\omega$.

For pump-probe delays longer than a few hundred femtoseconds, the thermalization of the electrons is achieved, thus leading to an increase of the electron temperature of several hundred degrees. However, the electronic distribution is not in thermal equilibrium with the lattice, the thermal relaxation to the lattice being achieved in a few picoseconds via electron-phonon scattering. The energy exchange between the electrons and the lattice can be described by the two temperature model leading to a time-dependent electron temperature $T_{e}(t)[63]$

$$
\begin{aligned}
C_{e} \frac{\partial T_{e}}{\partial t} & =-G\left(T_{e}-T_{i}\right)+P(t) \\
C_{i} \frac{\partial T_{i}}{\partial t} & =G\left(T_{e}-T_{i}\right)
\end{aligned}
$$

where $P(t)$ represents the laser source term, $C_{i}\left(C_{e}\right)$ is the lattice (electron) heat capacity, and $G$ is the electron-lattice coupling factor. In this simplified model, the two temperatures are assumed to be spatially uniform and therefore the heat propagation is neglected. 
Provided that the relative changes of the dielectric function with respect to a non-perturbed system are weak (linear regime) and that they are only due to a modification of the electron temperature, one may identify the spectral dependence of the differential transmission measured for a given time delay as the difference of the linear absorption cross-sections evaluated at different electron temperatures. More precisely, the differential transmission is expressed as

$$
\begin{aligned}
\frac{\Delta T}{T}(\tau, \omega) & =\frac{T\left[T_{e}(\tau), \omega\right]-T\left[T_{e}(0), \omega\right]}{T\left[T_{e}(0), \omega\right]}=-\Delta \tilde{\alpha}(\omega) l \\
& =\frac{3}{2 \pi R^{2}}\left[\sigma\left(\omega ; T_{e}(0)\right)-\sigma\left(\omega ; T_{e}(\tau)\right)\right]
\end{aligned}
$$

where $l=2 R$ is the sample thickness (here, the diameter of the nanoparticle), $T\left[T_{e}(\tau), \omega\right]$ and $T\left[T_{e}(0), \omega\right]$ are the probe transmissions in the presence and absence of the pump, respectively, and $\Delta \tilde{\alpha}$ is the pump-induced absorption change. Obviously $T\left[T_{e}(0), \omega\right]$ corresponds to an absorption at room temperature $T_{e}(0)=300 \mathrm{~K}$ for the conditions where the pump-probe experiments have been performed. We have computed the optical spectrum of a closed-shell

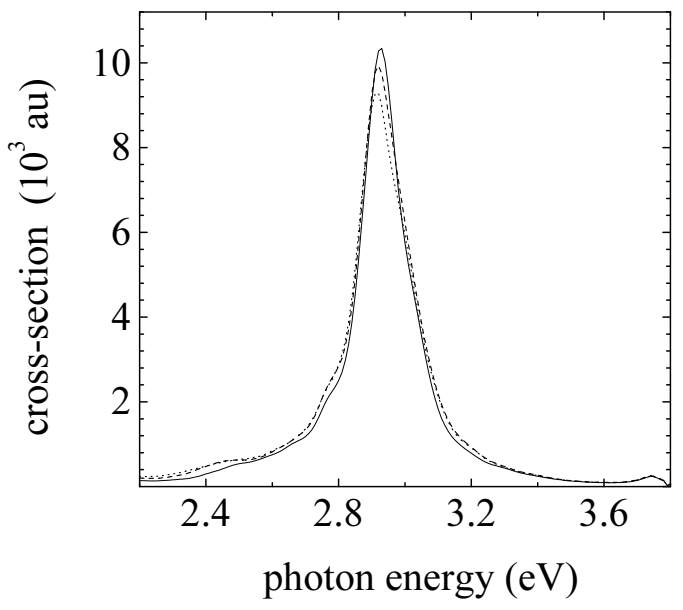

Fig. 2. TDLDA photoabsorption cross-section (in atomic units) of $\mathrm{Ag}_{2998}$ encapsulated in a transparent matrix $\left(\varepsilon_{m}=1.5\right)$ as a function of the photon energy. Solid line: $T_{e}=0 \mathrm{~K}$; dashed line: $T_{e}=300 \mathrm{~K}$; dotted line: $T_{e}=1200 \mathrm{~K}$.

nanoparticle $\mathrm{Ag}_{2998}$ embedded in a transparent matrix (alumina $\epsilon_{m}=1.5$ ) for three values of the temperature. The diameter of the nanoparticle is $4.6 \mathrm{~nm}$ and the photon energy ranges from $2.2 \mathrm{eV}$ to the interband threshold energy at $3.8 \mathrm{eV}$, i.e. in the spectral region associated to the surface plasmon of $\mathrm{Ag}$ nanoparticles. All these values correspond to typical experimental conditions performed in our group [6]. The results are presented in Fig. 2. The calculated 
oscillator strength is $90 \%$. Indeed, due to the presence of the surface plasmon resonance, almost all the oscillator strength is concentrated in this energy range. A clear red-shift and broadening of the resonance as a function of the electron temmperature is observed.

In the left panel of Fig. 3, the predictions of the normalized differential transmission [Eq. (30)] are presented as a function of the photon energy of the probe. The comparison is made for two electron temperatures $T_{e}=600 \mathrm{~K}$ and $T_{e}=1200 \mathrm{~K}$. The asymmetric shape of $\Delta T / T$ around the resonance energy is related to a combination of a red-shift and a broadening of the surface plasmon resonance. In the right panel of Fig. 3 the experimental spectrum of the normalized $\Delta T / T$ obtained for a pump-probe delay of $\tau=2 \mathrm{ps}$ is depicted. The pump pulse is set at $400 \mathrm{~nm}$ (second harmonic of a titanium sapphire laser amplified at $5 \mathrm{kHz}$ ) and the probe comes from a continuum generated in a sapphire cristal with the fundamental frequency of the amplified laser [6].

The asymmetric spectral shape of the differential transmission spectrum in Fig. 3, which is related to the shift and broadening of the plasmon, may have several origins. As pointed out in Refs. [6, 12,64], the interband transition induces a modification of the real part of the dielectric function in this spectral region, the resonance being far enough from the interband threshold to induce significant changes of the corresponding imaginary part. As stressed in Refs. $[12,64]$, this is a strong indication that intraband processes also play an important role. Indeed, as clearly seen in Fig. 2, the conduction electrons contribution leads both to a shift and to a broadening. We can therefore conclude that one needs to consider both the interband and intraband part on the same footing. Whereas this effect was previously taken into account in a phenomenological way via a shifted and broadened Lorentzian shape, here we have derived it directly from a quantum many-body approach based on the TDLDA at finite temperature.

\subsection{Phase space methods: from Hartree to Wigner and Vlasov}

As we have seen in Sec. 2.1, the most fundamental model for the quantum $N$-body problem is the Schrödinger equation for the $N$-particle wave function $\Psi\left(\boldsymbol{r}_{1}, \boldsymbol{r}_{2}, \ldots, \boldsymbol{r}_{N}, t\right)$. Unfortunately, the full Schrödinger equation cannot be solved exactly except for very small systems. A drastic, but useful and to some extent plausible, simplification can be achieved by neglecting two-body (and higher order) correlations. This amounts to assume that the $N$-body wave function can be factored into the product of $N$ one-body functions:

$$
\Psi\left(\boldsymbol{r}_{1}, \boldsymbol{r}_{2}, \ldots, \boldsymbol{r}_{N}, t\right)=\psi_{1}\left(\boldsymbol{r}_{1}, t\right) \psi_{2}\left(\boldsymbol{r}_{2}, t\right) \ldots \psi_{N}\left(\boldsymbol{r}_{N}, t\right) .
$$

For fermions, a weak form of the exclusion principle is satisfied if none of the wave functions on the right-hand side of Eq. (31) are identical ${ }^{5}$.

\footnotetext{
${ }^{5}$ A stronger version of the exclusion principle requires that $\Psi\left(\boldsymbol{r}_{1}, \boldsymbol{r}_{2}, \ldots, \boldsymbol{r}_{N}, t\right)$ is antisymmetric, i.e. that it changes sign when two of its arguments are inter-
} 

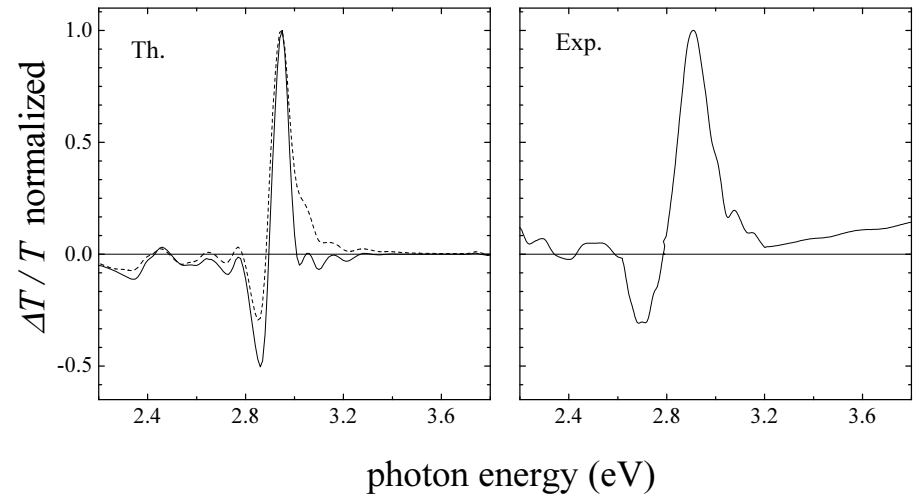

Fig. 3. Left panel: theoretical predictions of the normalized differential transmission for $\mathrm{Ag}_{2998}$ embedded in a transparent matrix as a function of the photon energy of the probe. Solid line: $T_{e}=600 \mathrm{~K}$; dotted line: $T_{e}=1200 \mathrm{~K}$. Right panel: Normalized experimental spectrum of $\Delta T / T$ of silver nanoparticles encapsulated in an alumina matrix for a pump-probe delay of 2 ps [6].

When the above assumption is made, the $N$-body Schrödinger equation reduces to a set of one-particle equations, coupled through Poisson's equation (time-dependent Hartree model):

$$
\begin{aligned}
i \hbar \frac{\partial \psi_{\alpha}}{\partial t} & =-\frac{\hbar^{2}}{2 m} \Delta \psi_{\alpha}-e \phi \psi_{\alpha}, \quad \alpha=1 \ldots N_{\text {orb }} \\
\Delta \phi & =\frac{e}{\varepsilon}\left(\sum_{\alpha=1}^{N_{\text {orb }}} p_{\alpha}\left|\psi_{\alpha}\right|^{2}-n_{i}(\boldsymbol{r})\right),
\end{aligned}
$$

where $N_{\text {orb }} \geq N$ is the number of occupied orbitals, $e$ and $m$ are the absolute electron charge and mass, and $\varepsilon$ is the dielectric constant; $n_{i}(\boldsymbol{r})$ is the ion density, which is supposed to be fixed and a continuous function of the position coordinate. This is known as the 'jellium' hypothesis and is valid whenever the relevant length scales are significantly larger that the ionic lattice spacing $a_{\text {latt }} \sim 5 \AA$. As mentioned in Sec. 2, this is the case for semiconductor nanostructures, but not so for metals (see Table 1 ); nevertheless, the jellium models still yields reasonably accurate results for all but the smallest nano-objects.

The occupation probabilities $p_{\alpha}\left(\sum_{\alpha=1}^{N_{\text {orb }}} p_{\alpha}=1\right)$ are defined to describe a Fermi-Dirac distribution at finite electron temperature, $p_{\alpha}=\left[1+\exp \left(\beta\left(\epsilon_{\alpha}-\right.\right.\right.$ $\mu)]^{-1}$, where $\beta=1 / k_{B} T_{e}, \mu$ is the chemical potential, and $\epsilon_{\alpha}$ is the singleparticle energy level. In practice, one first needs to obtain the ground-state equilibrium solution of Eqs. (32)-(33), which amounts to determining the $N_{\text {orb }}$

changed. This can be achieved by taking, instead of the single product of $N$ wave functions as in Eq. (31), a linear combinations of all products obtained by permutations of the arguments, with weights \pm 1 (Slater determinant) [65]. This is at the basis of Fock's generalization of the Hartree model 
occupation probabilities and the corresponding energy levels and wave functions. Subsequently, the equilibrium can be perturbed to study the electron dynamics. The numerical methods for the dynamics are quite standard, as the Eqs. (32) are basically one-particle Schrödinger equations. We will not enter into the details of the numerical methods in this paper: a list of relevant works on the Schrödinger equation can be found in Ref. [66].

We now show that the Hartree equations can be written in a completely equivalent form by making use of the Wigner transformation. The Wigner representation [29] is a useful tool to express quantum mechanics in a phase space formalism (for reviews see $[67,68,69]$ ). The Wigner function is a function of the phase space variables $(x, v)$ and time, which, in terms of the single-particle wave functions, reads as

$$
f(x, v, t)=\sum_{\alpha=1}^{N_{\text {orb }}} \frac{m}{2 \pi \hbar} p_{\alpha} \int_{-\infty}^{+\infty} \psi_{\alpha}^{*}\left(x+\frac{\lambda}{2}, t\right) \psi_{\alpha}\left(x-\frac{\lambda}{2}, t\right) e^{i m v \lambda / \hbar} d \lambda
$$

(we restrict our discussion to one-dimensional cases, but all results can easily be generalized to three dimensions). It must be stressed that the Wigner function, although it possesses many useful properties, is not a true probability density, as it can take negative values. However, it can be used to compute averages just like in classical statistical mechanics. For example, the expectation value of a generic quantity $A(x, v)$ is defined as:

$$
\langle A\rangle=\frac{\iint f(x, v) A(x, v) d x d v}{\iint f(x, v) d x d v},
$$

and yields the correct quantum-mechanical value ${ }^{6}$. In addition, the Wigner function reproduces the correct quantum-mechanical marginal distributions, such as the spatial density:

$$
n(x, t)=\int_{-\infty}^{+\infty} f(x, v, t) d v=\sum_{\alpha=1}^{N_{\text {orb }}} p_{\alpha}\left|\psi_{\alpha}\right|^{2} .
$$

We also point out that, of course, not all functions of the phase space variables are genuine Wigner functions, as they cannot necessarily be written in the form of Eq. (34). In general, although it is trivial to find the Wigner function given the wave functions that define the quantum mixture, the inverse operation is not generally feasible. Indeed, there are no simple rules to establish whether a given function of $x$ and $v$ is a genuine Wigner function. For a more detailed discussion on this issue, and some practical recipes to construct genuine Wigner functions, see [70].

\footnotetext{
${ }^{6}$ For variables whose corresponding quantum operators do not commute (such as $\hat{x} \hat{v})$, Eq. (35) must be supplemented by an ordering rule, known as Weyl's rule [69].
} 
The Wigner function obeys the following evolution equation:

$$
\begin{gathered}
\frac{\partial f}{\partial t}+v \frac{\partial f}{\partial x}+ \\
\frac{e m}{2 i \pi \hbar^{2}} \iint d \lambda d v^{\prime} e^{i m\left(v-v^{\prime}\right) \lambda / \hbar}\left[\phi\left(x+\frac{\lambda}{2}\right)-\phi\left(x-\frac{\lambda}{2}\right)\right] f\left(x, v^{\prime}, t\right)=0,
\end{gathered}
$$

where $\phi(x, t)$ is the self-consistent electrostatic potential obtained self-consistently from Poisson's equation (33).

Developing the integral term in Eq. (37) up to order $O\left(\hbar^{2}\right)$ we obtain

$$
\frac{\partial f}{\partial t}+v \frac{\partial f}{\partial x}+\frac{e}{m} \frac{\partial \phi}{\partial x} \frac{\partial f}{\partial v}=\frac{e \hbar^{2}}{24 m^{3}} \frac{\partial^{3} \phi}{\partial x^{3}} \frac{\partial^{3} f}{\partial v^{3}}+O\left(\hbar^{4}\right) .
$$

In the limit $\hbar \rightarrow 0$ one recovers the classical Vlasov equation, well-known from plasma physics (see Fig. 1). The Vlasov-Poisson system has been used to study the dynamics of electrons in metal clusters and thin metal films [45, 18]. It is appropriate for large excitation energies, for which the electrons' de Broglie wavelength is relatively small, thus reducing the importance of quantum effects in the electron dynamics. Nevertheless, for metallic nanostructures at room temperature, the equilibrium must be given by a Fermi-Dirac distribution, because the Fermi temperature is very high (see Table 1). For semiconductor nanostructures, $T_{F} \sim 10-50 \mathrm{~K}$, so that a Maxwell-Boltzmann equilibrium is sometimes appropriate for moderate temperatures.

The Wigner equation must be coupled to the Poisson's equation for the electric potential:

$$
\frac{\partial^{2} \phi}{\partial x^{2}}=-\frac{e}{\varepsilon}\left[n_{i}(x)-n(x, t)\right],
$$

The resulting Wigner-Poisson (WP) system has been extensively used in the study of quantum transport [71, 72, 73]. Exact analytical results can be obtained by linearizing Eqs. (37) and (39) around a spatially homogeneous equilibrium given by $n_{0} f_{0}(v)$ (Maxwell-Boltzmann or Fermi-Dirac distribution), where $n_{0}=n_{i}=$ const. is the uniform equilibrium density. By expressing the fluctuating quantities as a sum of plane waves $\exp (i k x-i \omega t)$ with frequency $\omega$ and wave number $k$, the dispersion relation can be written in the form $\varepsilon(k, \omega)=0$, where the 'dielectric constant' $\varepsilon$ reads, for the WP system,

$$
\varepsilon_{\mathrm{WP}}(\omega, k)=1+\frac{m \omega_{p}^{2}}{n_{0} k} \int \frac{f_{0}(v+\hbar k / 2 m)-f_{0}(v-\hbar k / 2 m)}{\hbar k(\omega-k v)} d v
$$

or equivalently

$$
\varepsilon_{\mathrm{WP}}(\omega, k)=1-\frac{\omega_{p}^{2}}{n_{0}} \int \frac{f_{0}(v)}{(\omega-k v)^{2}-\hbar^{2} k^{4} / 4 m^{2}} d v .
$$


This is just the Lindhard [74] dispersion relation, well known from solid-state physics. From Eq. (40), one can recover the Vlasov-Poisson dispersion relation by taking the classical limit $\hbar \rightarrow 0$

$$
\varepsilon_{\mathrm{VP}}(\omega, k)=1+\frac{\omega_{p}^{2}}{n_{0} k} \int \frac{\partial f_{0} / \partial v}{\omega-k v} d v
$$

The equivalence of the Hartree and Wigner-Poisson methods can be easily proven by comparing the linear results. For the Hartree equations (32), we linearize around a homogeneous equilibrium given by plane waves:

$$
\psi_{\alpha}=\sqrt{n_{0}} \exp \left(i \frac{m u_{0 \alpha}}{\hbar} x\right),
$$

each with occupation number $p_{\alpha}$ and energy $\epsilon_{\alpha}=m u_{0 \alpha}^{2} / 2$. The Hartree dielectric constant is found to be

$$
\varepsilon_{\mathrm{H}}(\omega, k)=1-\sum_{\alpha=1}^{N_{\text {orb }}} p_{\alpha} \frac{\omega_{p}^{2}}{\left(\omega-k u_{0 \alpha}\right)^{2}-\hbar^{2} k^{4} / 4 m^{2}},
$$

which is a discrete form of the Wigner-Poisson dispersion relation (41).

Example - Ultrafast electron dynamics in thin metal films

Several experiments have shown $[2,3]$ that electron transport in thin metal films occurs on a femtosecond time scale and involves ballistic electrons traveling at the Fermi velocity of the metal $v_{F}$. More recently, a regime of lowfrequency nonlinear oscillations (corresponding to ballistic electrons bouncing back and forth on the film surfaces) was measured in transient reflection experiments on thin gold films [75].

These findings were corroborated by accurate numerical simulations based on the one-dimensional Vlasov-Poisson equations [18]. The electrons are initially prepared in a Fermi-Dirac equilibrium at finite (but small) temperature. They are subsequently excited by imposing a constant velocity shift $\Delta v=0.08 v_{F}$ to the initial distribution, which is a rather strong excitation. This scenario is appropriate when no linear momentum is transferred parallel to the plane of the surface (i.e., $q_{\|}=0$ ) and is relevant to the excitation of the film with optical pulses [76]. For $q_{\|}=0$, only longitudinal modes (volume plasmon with $\omega=\omega_{p}$ ) can be excited.

As a reference case, we studied a sodium film with initial temperature $T_{e}=$ $0.008 T_{F} \simeq 300 \mathrm{~K}$ and thickness $L \simeq 120 \AA$. The time evolution of the thermal $E_{\mathrm{th}}$ and center-of-mass $E_{\mathrm{cm}}$ energies was analyzed (Fig. 4). During an initial rapidly-oscillating phase, $E_{\mathrm{cm}}$ is almost entirely converted into thermal energy (Landau damping). After saturation, a slowly oscillating regime appears, with period equal to $50 \omega_{p}^{-1} \approx 5.3 \mathrm{fs}$, where $\omega_{p}=\left(e^{2} n / m \varepsilon_{0}\right)^{1 / 2}$ is the plasmon frequency. This period is close to the time of flight of electrons traveling at 


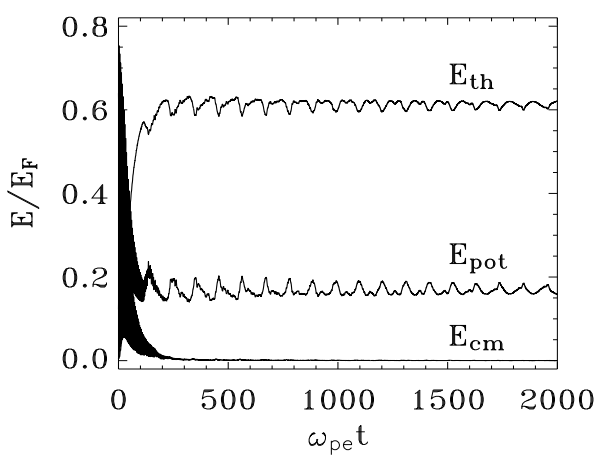

Fig. 4. Time evolution of the thermal, potential, and center-of-mass energies of the electron population in a thin sodium film.

the Fermi velocity and bouncing back and forth on the film surfaces (further details are provided in our previous work [18]).

The phase space portrait of the electron distribution, shown in Fig. 5 clearly reveals that the perturbation starts at the film surfaces, and then proceeds inward at the Fermi velocity of the metal. The structure formation at the Fermi surface, which has spread over the entire film for $\omega_{p} t>150$, is responsible for the increase of the thermal energy (and thus the electron temperature) observed in Fig. 4. As no coupling to an external environment (e.g., phonons) is present, this excess temperature cannot be dissipated.
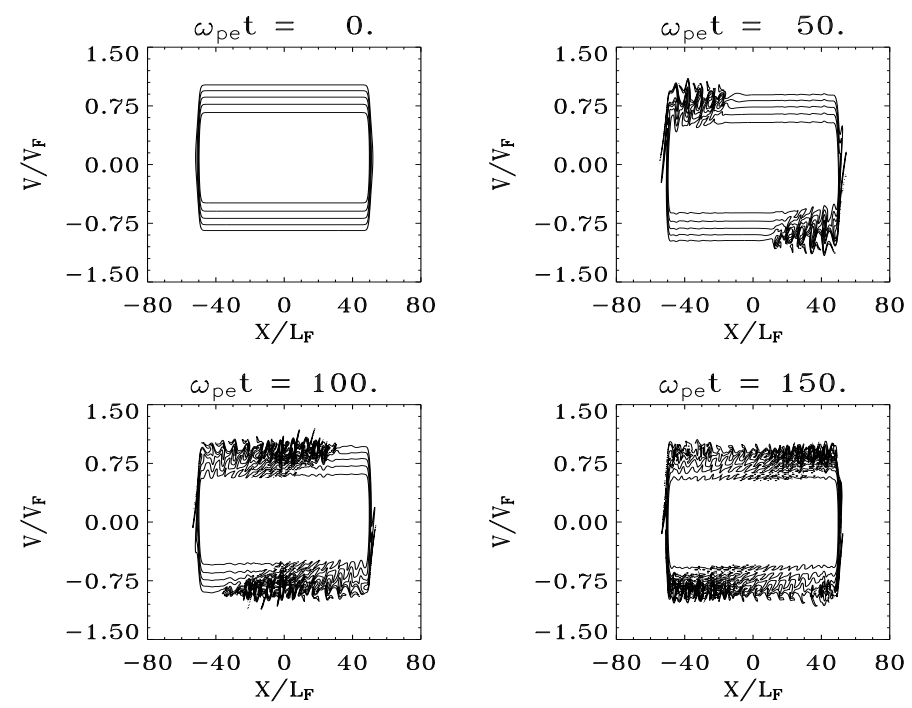

Fig. 5. Phase space portrait of the electron distribution. Velocity is normalized to the Fermi velocity, and space to the Thomas-Fermi screening length $L_{F}=v_{F} / \omega_{p}$. 
Quantum simulations of the electron dynamics using the Wigner-Poisson system were performed more recently: as expected, the Vlasov results were recovered in the large excitation regime $\delta v>0.08 v_{F}$. For smaller excitations, a different regime appears, in which the ballistic oscillations described above are no longer observed. Further work is in progress on this issue [77].

\section{Beyond the mean field}

The mean-field approach described above is appropriate to describe the electron dynamics on very short time scales $(<100 \mathrm{fs})$. On a longer time scale $(0.1-$ $1 \mathrm{ps})$, the injected energy is redistributed among the electrons via electronelectron (e-e) collisions. Electron-phonon (e-ph) thermalization (i.e., coupling to the ionic lattice) is generally supposed to occur on even longer time scales. However, the results of Refs. [5, 78] on thin gold films have shown that nonequilibrium electrons start interacting with the lattice earlier than expected, so that a clear-cut separation between e-e and e-ph relaxation is not entirely pertinent.

The phase-space approach is particularly well-suited to include corrections that go beyond the mean-field picture. This can be done with relative ease for semiclassical models (Vlasov), by using a Boltzmann-like e-e collision integral that respects Pauli's exclusion principle (Ühling-Uhlenbeck model) [79]:

$$
\left(\frac{\partial f}{\partial t}\right)_{\mathrm{UU}}=\int \frac{d^{3} \mathbf{p}_{\mathbf{2}} d \Omega}{(2 \pi \hbar)^{3}} \sigma(\Omega)\left|v_{12}\right|\left(f_{1} f_{2} \bar{f}_{3} \bar{f}_{4}-f_{3} f_{4} \bar{f}_{1} \bar{f}_{2}\right)
$$

where $v_{12}$ is the relative velocity of the colliding particles 1 and $2, \sigma(\Omega)$ is the differential cross section depending on the scattering angle $\Omega$, and indices 3 and 4 label the outgoing momenta, $f_{i}=f\left(\boldsymbol{r}, \boldsymbol{p}_{i}, t\right)$ and $\bar{f}_{i}=1-f_{i} / 2$. This collision term is similar to the well known classical Boltzmann collision term but for Pauli blocking factors $\bar{f}_{i} \bar{f}_{j}$. As known from solid-state physics, this blocking factor plays a dramatic role for electronic systems [65]. At $T_{e}=$ $0 \mathrm{~K}$, all collisions are Pauli blocked and the collisional mean-free path of the electrons becomes infinite. But if the system becomes excited, phase space opens up and activates the collision term. The effect of the above e-e collision term on the semiclassical Vlasov dynamics in metal clusters was investigated numerically in [80].

It is conceptually harder to include collisions in fully quantum models. A significant constraint is that nonunitary corrections to the Wigner equation should be written in 'Lindblad form' [81], which guarantees that the evolved Wigner function corresponds to a positive-definite density matrix.

The Ühling-Uhlenbeck collision term (45) is a complicated nonlinear integral, which is difficult to implement in a numerical code. It is therefore useful to construct some simplified collision terms that are more easily amenable to numerical treatment. In the following, we briefly illustrate two simple models of e-e and e-ph collisions that we have employed in our previous works. 
Electron-electron collisions.

To model e-e collisions, a relaxation term is added to the right-hand side of the Vlasov or Wigner equation:

$$
\left(\frac{\partial f}{\partial t}\right)_{\mathrm{e}-\mathrm{e}} \equiv-\nu_{e e}\left(T_{e}\right)\left(f-f_{\infty}\right),
$$

where $\nu_{e e}$ is the average e-e collision rate and $f_{\infty}(x, v)$ is a Fermi-Dirac distribution. The idea behind this model is that the electron distribution will eventually relax, on a time scale of the order $\nu_{e e}^{-1}$, towards a Fermi-Dirac equilibrium $f_{\infty}$ with total energy equal to that of the initial electron distribution $f\left(x, v, t=0^{+}\right)$, including of course the initial excitation energy. For electrons near the Fermi surface, the e-e collision rate can be written as [82]

$$
\nu_{e e}\left(T_{e}\right)=a\left(k_{B} T_{e}\right)^{2},
$$

where $a$ is a (dimensional) proportionality constant. The latter has been estimated from numerical simulations of the electron dynamics in sodium clusters [80], yielding $a \simeq 0.4 \mathrm{fs}^{-1} \mathrm{eV}^{-2}$, which is also compatible with the analytical prediction given by the random phase approximation [82]. The electron temperature is computed instantaneously during the simulation, and plugged into the expression for the collision rate (47). It is important to underline that the above model for e-e collisions, though simple, is completely self-contained and requires no additional $a d-h o c$ parameters. The model has been applied to the electron dynamics in thin metal films. The slow ballistic oscillations of Fig. 4 are still observed, although they are damped on a time scale of the order of $500 \omega_{p e}^{-1} \simeq 50 \mathrm{fs}$ (see Fig. 6).

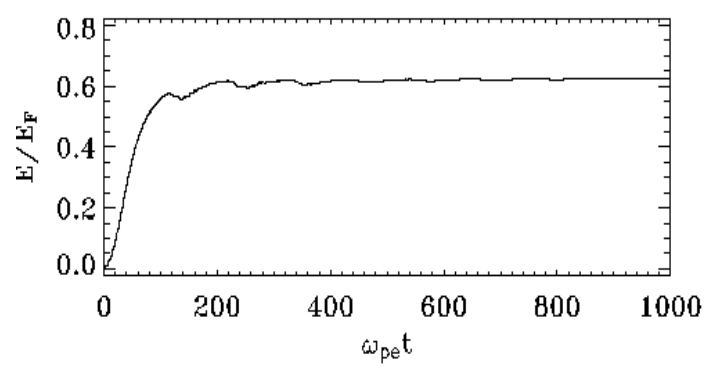

Fig. 6. Evolution of the thermal energy for a case with e-e collisions and $L=$ $100 L_{F} \simeq 120 \AA$.

\section{Electron-phonon collisions.}

By coupling to the ionic lattice, the electrons progressively relax to a thermal distribution with a temperature equal to that of the lattice $T_{i}$. This relaxation 
time is generally termed $\tau_{1}$ in the semiconductor literature. In addition, the lattice acts as an external environment for the electrons, leading to a loss of quantum coherence over a time scale $\tau_{2}$ (decoherence time). The relaxation and decoherence times correspond, respectively, to the decay of diagonal and nondiagonal terms in the density matrix describing the electron population.

Such environment-induced decoherence can be modeled, in the Wigner representation, by an appropriate friction-diffusion term [83]:

$$
\left(\frac{\partial f}{\partial t}\right)_{\mathrm{e}-\mathrm{ph}}=2 \gamma \frac{\partial(v f)}{\partial v}+D_{v} \frac{\partial^{2} f}{\partial v^{2}}+D_{x} \frac{\partial^{2} f}{\partial x^{2}},
$$

where $\gamma$ is the relaxation rate (inverse of the relaxation time $\tau_{1}$ ), and $D_{v}$, $D_{x}$ are diffusion coefficients in velocity and real space respectively, which are related to the decoherence time $\tau_{2}$ and depend on the lattice temperature $T_{i}$. The effect of the diffusive terms is to smooth out the fine structure of the Wigner function, thus suppressing interference phenomena, which are a typically quantum effect. Finally, we recall that, in order to preserve the positivity of the density matrix associated to the Wigner distribution function, the eph collision term (48) must be in Lindblad form [81]. This is automatically achieved [84] if the coefficients respect the inequality $D_{v} D_{x} \geq \gamma^{2} \hbar^{2} / 4 m^{2}$.

\subsection{Hydrodynamical models: from micro to macro}

Despite its considerable interest, the Wigner-Poisson (WP) formulation presents some intrinsic drawbacks : (i) it is a nonlocal, integro-differential system; and (ii) its numerical treatment requires the meshing of the whole phase space. Moreover, as is often the case with kinetic models, the Wigner-Poisson system gives more information than one is really interested in. For these reasons, it would be useful to obtain an accurate reduced model which, though not providing the same detailed information, is still able to reproduce the main features of the physical system under consideration.

In this section, we will derive an effective Schrödinger-Poisson (SP) system, which, in an appropriate limit, reproduces the results of the kinetic WP formulation [85]. In order to obtain the effective SP system, we will first derive a system of reduced hydrodynamic (or fluid) equations by taking moments of the WP system. It will be shown that the pressure term appearing in the fluid equations can be decomposed into a classical and a quantum part. With some reasonable hypotheses on the pressure term, the fluid system can be closed. For simplicity of notation, only one-dimensional problems will be considered, but the results can be easily extended to higher dimensions.

In order to derive a fluid model, we take moments of Eq. (37) by integrating over velocity space. Introducing the standard definitions of density, mean velocity, and pressure

$$
n=\int f d v, \quad u=\frac{1}{n} \int f v d v, \quad P=m\left(\int f v^{2} d v-n u^{2}\right),
$$


it is obtained

$$
\begin{aligned}
& \frac{\partial n}{\partial t}+\frac{\partial(n u)}{\partial x}=0, \\
& \frac{\partial u}{\partial t}+u \frac{\partial u}{\partial x}=\frac{e}{m} \frac{\partial \phi}{\partial x}-\frac{1}{m n} \frac{\partial P}{\partial x} .
\end{aligned}
$$

We immediately notice that, surprisingly, Eqs. (50)-(51) do not differ from the ordinary evolution equations for a classical fluid. It can be shown, however, that quantum effects are actually hidden in the pressure term, which may be decomposed into a classical and a quantum part.

By using the definition of the Wigner function (34) and representing each state in terms of its amplitude $\sqrt{n_{\alpha}}$ and phase $S_{\alpha}$

$$
\psi_{\alpha}(x, t)=\sqrt{n_{\alpha}(x, t)} \exp \left(i S_{\alpha}(x, t) / \hbar\right),
$$

we obtain that $P=P^{C}+P^{Q}$. The classical part of the pressure can be written as

$$
P^{C}=m n\left[\sum_{\alpha} p_{\alpha} \frac{n_{\alpha}}{n} u_{\alpha}^{2}-\left(\sum_{\alpha} p_{\alpha} \frac{n_{\alpha}}{n} u_{\alpha}\right)^{2}\right] \equiv m n\left(\left\langle u_{\alpha}^{2}\right\rangle-\left\langle u_{\alpha}\right\rangle^{2}\right),
$$

where $m u_{\alpha}=\partial S_{\alpha} / \partial x$ [the $u_{\alpha}$ 's should not be mistaken with the global mean velocity $u$ defined in Eq. (49)]. This is the standard expression for the pressure as velocity dispersion, thus justifying the term 'classical' pressure.

The quantum part of the pressure is written as

$$
P^{Q}=\frac{\hbar^{2}}{2 m} \sum_{\alpha} p_{\alpha}\left(\left(\frac{\partial \sqrt{n_{\alpha}}}{\partial x}\right)^{2}-\sqrt{n_{\alpha}} \frac{\partial^{2} \sqrt{n_{\alpha}}}{\partial x^{2}}\right) .
$$

It can be shown that, for distances larger that the Thomas-Fermi screening length $L_{F}$, one can replace $n_{\alpha}$ with $n$, the total density as defined in Eq. (49). In order to close the fluid system (50)-(51) one still has to express the classical pressure in terms of the density $n$. This is the standard procedure adopted in classical hydrodynamics: the relation $P^{C}(n)$ is the equation of state, and depends on the particular conditions of the system, notably its temperature.

With these hypotheses, the Eq. (51) reduces to

$$
\frac{\partial u}{\partial t}+u \frac{\partial u}{\partial x}=\frac{e}{m} \frac{\partial \phi}{\partial x}-\frac{1}{m} \frac{\partial W}{\partial x}+\frac{\hbar^{2}}{2 m^{2}} \frac{\partial}{\partial x}\left(\frac{\partial^{2}(\sqrt{n}) / \partial x^{2}}{\sqrt{n}}\right),
$$

where we have defined the effective potential

$$
W(n)=\int^{n} \frac{d n^{\prime}}{n^{\prime}} \frac{d P^{C}\left(n^{\prime}\right)}{d n^{\prime}} .
$$

Equations (50) and (55) constitute the quantum hydrodynamical approximation to the full Wigner (or Hartree) equation. 
It is now possible to combine Eqs. (50) and (55) into an effective nonlinear Schrödinger equation. To this purpose, let us define the effective wavefunction

$$
\Psi=\sqrt{n(x, t)} \exp (i S(x, t) / \hbar),
$$

with $S(x, t)$ defined according to $m u(x, t)=\partial S(x, t) / \partial x$. We obtain that $\Psi(x, t)$ satisfies the equation

$$
i \hbar \frac{\partial \Psi}{\partial t}=-\frac{\hbar^{2}}{2 m} \frac{\partial^{2} \Psi}{\partial x^{2}}-e \phi \Psi+W \Psi .
$$

By linearizing Eqs. (50) and (55) around a homogeneous equilibrium, we obtain the following dispersion relation

$$
\omega^{2}=\omega_{p}^{2}+v_{0}^{2} k^{2}+\frac{\hbar^{2} k^{4}}{4 m^{2}},
$$

where $m v_{0}^{2}=\left(d P^{C} / d n\right)_{n=n_{0}}$. It can be proven that, by an appropriate choice of the equation of state $P^{C}(n)$, Eq. (59) reproduces correctly the leading terms of the Hartree or Wigner dispersion relation.

To summarize, we have shown that, under appropriate conditions, the Hartree or Wigner models can be reduced to a set of two hydrodynamical equations (50) and (55), or, equivalently, to a single nonlinear Schrödinger equation (58). The two hypotheses used for this reduction were that (i) all quantities vary on a length scale larger than $L_{F}$; and (ii) the equation of state for the classical pressure is $P^{C}=P^{C}(n)$ (standard fluid closure).

\section{Example — Thin metal films}

We have studied the electron dynamics in a thin metal film using the above quantum hydrodynamical model [86]. A preliminary result is shown in Fig. 7 , where we plot the evolution of the thermal and potential energies against time. In order to compare to the Vlasov simulations described in Sec. 2.3, the hydrodynamic equations are solved in the semiclassical limit, i.e. using a small value of the Planck constant normalized to $E_{F} / \omega_{p}$ (note however that here the initial excitation $\delta v=0.22 v_{F}$ is larger compared to the case of Fig. 4 , where $\delta v=0.08 v_{F}$ ). The hydrodynamic results display some coherent oscillations at high frequency, which are a typical signature of quantum effects. Nevertheless, the initial increase of the thermal energy is clearly captured and the subsequent ballistic oscillations are still visible, particularly on the potential energy.

\section{Spin dynamics}

The dynamics of magneto-optical processes in metallic nanostructures depends on the temporal and spatial characteristics that are being investigated. 

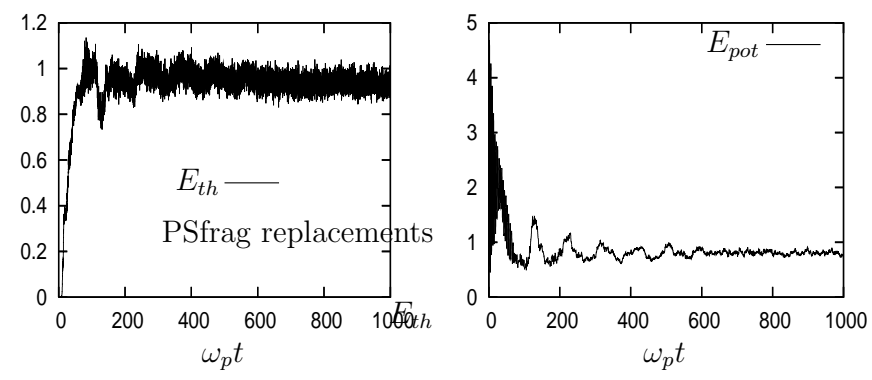

Fig. 7. Time evolution of the thermal and potential energies (normalized to $E_{F}$ ) of the electron population, obtained using a quantum hydrodynamics model.

Short time scale $\left(t<10^{-12} \mathrm{~s}\right)$ have only been explored recently. In 1996, the group of Jean-Yves Bigot in Strasbourg highlighted the existence of ultrafast demagnetization processes (within less than a hundred femtoseconds) induced by femtosecond laser pulses in ferromagnetic thin films [24]. These demagnetization processes are not yet fully understood.

From a theoretical point of view, very little is known on the time-dependent magneto-optical response of metallic nanostructures to an ultrafast optical pulse. The main difficulty is to provide an adequate description of the interplay between electronic and spin degrees of freedom in the metal. So far, only two theoretical models have been proposed to explain this effect $[25,26]$. These works are based on two different mechanisms: in [25], the spin-orbit coupling is invoked, whereas in [26] phonon or impurity mediated spin-flip scattering is privileged. Unfortunately, the parameters employed in [25] are not realistic and the model developed in [26] is a phenomenological approach that does not allow quantitative predictions. From the above considerations it follows that there is a need for the development of efficient theoretical models able to explain in a quantitative manner the experimental findings.

A proper treatment of spin dynamics requires an extension of our model (TDLDA) to include spin degrees of freedom. In the following, the formalism of the time-dependent local-spin-density approximation (TDLSDA) in the linear regime (including also its extension to finite temperature) is presented. A second part will be devoted to the nonlinear dynamics.

\subsection{Linear response: local spin density approximation}

The generalization of the linear TDLDA to spin polarized electron systems has been performed by Rajagopal [87]. In the following we provide the basic equations of this approach including its extension to finite temperature.

Within the framework of DFT one can calculate the spin density matrix $n_{\sigma \sigma^{\prime}}(\boldsymbol{r})$ defined as 


$$
n_{\sigma \sigma^{\prime}}(\boldsymbol{r})=\left\langle 0\left|\hat{\psi}_{\sigma}^{+}(\boldsymbol{r}) \hat{\psi}_{\sigma^{\prime}}(\boldsymbol{r})\right| 0\right\rangle
$$

where $\hat{\psi}_{\sigma}^{+}(\boldsymbol{r})$ and $\hat{\psi}_{\sigma}(\boldsymbol{r})$ are the wave field operators corresponding to the creation and annihilation of an electron with spin $\sigma$ at position $r$, and $|0\rangle$ is the ground state of the system. When the system is subjected to a small local spindependent external potential $\delta V_{e x t}^{\sigma \sigma^{\prime}}(\boldsymbol{r} ; \omega)$ (this quantity describes the coupling of the charge and spin of the electrons to external electric and magnetic fields) the spin-density response function is defined through the equation

$$
\delta n_{\sigma \sigma^{\prime}}(\boldsymbol{r} ; \omega)=\sum_{\sigma_{1} \sigma_{2}} \int \chi_{\sigma \sigma^{\prime}, \sigma_{1} \sigma_{2}}\left(\boldsymbol{r}, \boldsymbol{r}^{\prime} ; \omega\right) \delta V_{e x t}^{\sigma_{1} \sigma_{2}}\left(\boldsymbol{r}^{\prime} ; \omega\right) d \boldsymbol{r}^{\prime}
$$

For the sake of simplicity, we restrict ourself to the case of collinear magnetism, i.e. to the case of a uniform direction of magnetization. This restriction leads to a diagonal spin-density matrix $\left(n_{\sigma \sigma^{\prime}}=n_{\sigma} \delta_{\sigma \sigma^{\prime}}\right)$ and simplified expressions. The spin-density response function defined in Eq. (61) reduces to

$$
\delta n_{\sigma}(\boldsymbol{r} ; \omega)=\sum_{\sigma^{\prime}} \int \chi_{\sigma \sigma^{\prime}}\left(\boldsymbol{r}, \boldsymbol{r}^{\prime} ; \omega\right) \delta V_{e x t}^{\sigma^{\prime}}\left(\boldsymbol{r}^{\prime} ; \omega\right) d \boldsymbol{r}^{\prime}
$$

which can be rewritten as

$$
\delta n_{\sigma}(\boldsymbol{r} ; \omega)=\sum_{\sigma^{\prime}} \int \chi_{\sigma \sigma^{\prime}}^{0}\left(\boldsymbol{r}, \boldsymbol{r}^{\prime} ; \omega\right) \delta V_{e f f}^{\sigma^{\prime}}\left(\boldsymbol{r}^{\prime} ; \omega\right) d \boldsymbol{r}^{\prime}
$$

with

$$
\begin{aligned}
\delta V_{e f f}^{\sigma}(\boldsymbol{r} ; \omega) & =\delta V_{e x t}^{\sigma}(\boldsymbol{r} ; \omega) \\
& +\sum_{\sigma^{\prime}} \int\left\{\frac{e^{2} / 4 \pi \epsilon_{0}}{\left|\boldsymbol{r}-\boldsymbol{r}^{\prime}\right|}+f_{x c}^{\sigma \sigma^{\prime}}\left(\boldsymbol{r}, \boldsymbol{r}^{\prime} ; \omega\right)\right\} \delta n_{\sigma^{\prime}}\left(\boldsymbol{r}^{\prime} ; \omega\right) d \boldsymbol{r}^{\prime}
\end{aligned}
$$

In the above expression the function $f_{x c}^{\sigma \sigma^{\prime}}\left(\boldsymbol{r}, \boldsymbol{r}^{\prime} ; \omega\right)$ is the Fourier transform of the time-dependent kernel defined by $f_{x c}^{\sigma \sigma^{\prime}}\left(\boldsymbol{r}, t ; \boldsymbol{r}^{\prime}, t^{\prime}\right) \equiv \delta V_{x c}^{\sigma}(\boldsymbol{r}, t) / \delta n_{\sigma^{\prime}}\left(\boldsymbol{r}^{\prime}, t^{\prime}\right)$ and $\chi_{\sigma \sigma^{\prime}}^{0}\left(\boldsymbol{r}, \boldsymbol{r}^{\prime} ; \omega\right)$ is the non-interacting retarded spin-density correlation function. For spin polarized electron systems the exchange-correlation potential is defined as

$$
V_{x c}^{\sigma}(\boldsymbol{r})=\left[\frac{\partial}{\partial n_{\sigma}}\left\{n \omega_{x c}\left(n_{+}, n_{-}\right)\right\}\right]_{n_{+}=n_{+}(\boldsymbol{r}) ; n_{-}=n_{-}(\boldsymbol{r})},
$$

where $\Omega_{x c}\left[n_{+}, n_{-}\right]=\int n(\boldsymbol{r}) \omega_{x c}\left(n_{+}(\boldsymbol{r}), n_{-}(\boldsymbol{r})\right) d \boldsymbol{r}$ is the exchange-correlation thermodynamic potential and $\omega_{x c}$ the exchange-correlation thermodynamic potential per particle of the homogeneous electron gas calculated at the local density $n$ and magnetization $m=n_{+}-n_{-}$. By noting that

$$
\frac{\partial}{\partial n_{\sigma}}\left\{n \omega_{x c}\left(n_{+}, n_{-}\right)\right\}=\frac{\partial}{\partial n}\left\{n \omega_{x c}(n, m)\right\}+\sigma \frac{\partial}{\partial m}\left\{n \omega_{x c}(n, m)\right\},
$$


the expression (65) can be rewritten as [88]

$$
V_{x c}^{\sigma}(\boldsymbol{r})=\left[\frac{\partial}{\partial n}\left\{n \omega_{x c}(n, m)\right\}\right]_{n=n(\boldsymbol{r}) ; m=m(\boldsymbol{r})}+\sigma \mu_{B} B_{x c}(\boldsymbol{r})
$$

where $B_{x c}(\boldsymbol{r})=\mu_{B}^{-1}\left[\frac{\partial}{\partial m}\left\{n \omega_{x c}(n, m)\right\}\right]_{n=n(\boldsymbol{r}) ; m=m(\boldsymbol{r})}$ is the exchange-correlation magnetic field acting on spin, and $\mu_{B}=e \hbar /(2 m)$ is the Bohr magneton. This is an internal magnetic field. The response functions $\chi^{0}$ and $\chi$ are related by an integral equation (to be more precise, due to the spin degree of freedom, it is a matrix integral equation)

$$
\begin{aligned}
\chi_{\sigma \sigma^{\prime}}\left(\boldsymbol{r}, \boldsymbol{r}^{\prime} ; \omega\right) & =\chi_{\sigma \sigma^{\prime}}^{0}\left(\boldsymbol{r}, \boldsymbol{r}^{\prime} ; \omega\right)+\sum_{\sigma_{1} \sigma_{2}} \iint \chi_{\sigma \sigma_{1}}^{0}\left(\boldsymbol{r}, \boldsymbol{r}^{\prime \prime} ; \omega\right) \\
& \times K^{\sigma_{1} \sigma_{2}}\left(\boldsymbol{r}^{\prime \prime}, \boldsymbol{r}^{\prime \prime \prime} ; \omega\right) \chi_{\sigma_{2} \sigma^{\prime}}\left(\boldsymbol{r}^{\prime \prime \prime}, \boldsymbol{r}^{\prime} ; \omega\right) d \boldsymbol{r}^{\prime \prime} d \boldsymbol{r}^{\prime \prime \prime},
\end{aligned}
$$

with the residual interaction defined by

$$
K^{\sigma_{1} \sigma_{2}}\left(\boldsymbol{r}, \boldsymbol{r}^{\prime} ; \omega\right)=\frac{e^{2}}{4 \pi \epsilon_{0}\left|\boldsymbol{r}-\boldsymbol{r}^{\prime}\right|} \delta_{\sigma_{1} \sigma_{2}}+f_{x c}^{\sigma_{1} \sigma_{2}}\left(\boldsymbol{r}, \boldsymbol{r}^{\prime} ; \omega\right) .
$$

As for TDLDA, in the adiabatic local-density approximation (ALDA) the exchange-correlation kernel is frequency-independent and local and reduces to

$$
f_{x c}^{\sigma \sigma^{\prime}}\left(\boldsymbol{r}, \boldsymbol{r}^{\prime}\right)=\left[\frac{\partial^{2}\left[n \omega_{x c}(n, m)\right]}{\partial n_{\sigma} \partial n_{\sigma^{\prime}}}\right]_{n=n(\boldsymbol{r}) ; m=m(\boldsymbol{r})} \delta\left(\boldsymbol{r}-\boldsymbol{r}^{\prime}\right) .
$$

It should be mentioned that the functional $\omega_{x c}$ in the above expression should be the same as the one used in the calculation of the ground state (see Eq. (65)). By using the same field-theory techniques employed previously for TDLDA (see Sec. 2.2), one can show that the free response function reads

$$
\begin{aligned}
\chi_{\sigma \sigma^{\prime}}^{0}\left(\boldsymbol{r}, \boldsymbol{r}^{\prime} ; \omega ; T_{e}\right) & =\delta_{\sigma \sigma^{\prime}} \sum_{k} f_{k}^{\sigma} \phi_{k}^{\sigma *}(\boldsymbol{r}) \phi_{k}^{\sigma}\left(\boldsymbol{r}^{\prime}\right) G_{+}^{\sigma}\left(\boldsymbol{r}, \boldsymbol{r}^{\prime} ; \varepsilon_{k}^{\sigma}+\hbar \omega ; T_{e}\right) \\
& +\sum_{k} f_{k}^{\sigma} \phi_{k}^{\sigma}(\boldsymbol{r}) \phi_{k}^{\sigma *}\left(\boldsymbol{r}^{\prime}\right) G_{+}^{\sigma *}\left(\boldsymbol{r}, \boldsymbol{r}^{\prime} ; \varepsilon_{k}^{\sigma}-\hbar \omega ; T_{e}\right)
\end{aligned}
$$

where $\phi_{k}^{\sigma}(\boldsymbol{r})$ and $\varepsilon_{k}^{\sigma}$ are the one-electron Kohn-Sham wave functions and energies, respectively. $G_{+}^{\sigma}$ is the one-particle retarded Green's function for the spins $\sigma$ and $f_{k}^{\sigma}=\left[1+\exp \left\{\left(\varepsilon_{k}^{\sigma}-\mu\right) / k_{B} T_{e}\right\}\right]^{-1}$. Similarly to TDLDA, we have assumed that the residual interaction (68) is temperature independent. Thus, it is consistent with the use of $\omega_{x c}(n, m)=\epsilon_{x c}(n, m)$ in the calculation of the ground-state properties.

From the above formalism one can compute the dipolar absorption crosssection

$$
\sigma\left(\omega ; T_{e}\right)=\frac{\omega}{\varepsilon_{0} c} \operatorname{Im}\left[\alpha\left(\omega ; T_{e}\right)\right],
$$


where $\alpha$ is the frequency-dependent dipole electric polarizability defined as

$$
\alpha\left(\omega ; T_{e}\right)=\int\left[\delta n_{+}\left(\boldsymbol{r} ; \omega ; T_{e}\right)+\delta n_{-}\left(\boldsymbol{r} ; \omega ; T_{e}\right)\right] \delta V_{e x t}(\boldsymbol{r} ; \omega) d \boldsymbol{r} .
$$

By analogy, one defines a quantity which is constructed from the local magnetization (instead of the local density)

$$
\sigma_{m}\left(\omega ; T_{e}\right)=\frac{\omega}{\varepsilon_{0} c} \operatorname{Im}\left[\alpha_{m}\left(\omega ; T_{e}\right)\right],
$$

where $\alpha_{m}$ is the frequency-dependent dipole magnetic polarizability defined as

$$
\alpha_{m}\left(\omega ; T_{e}\right)=\int\left[\delta n_{+}\left(\boldsymbol{r} ; \omega ; T_{e}\right)-\delta n_{-}\left(\boldsymbol{r} ; \omega ; T_{e}\right)\right] \delta V_{e x t}(\boldsymbol{r} ; \omega) d \boldsymbol{r} .
$$

On can show that $\sigma_{m}$ fulfils the following sum rule

$$
\int \sigma_{m}\left(\omega ; T_{e}\right) d \omega=\frac{2 \pi^{2} M\left(T_{e}\right)}{c}
$$

where $M=N^{+}-N^{-}$is the total magnetization of the system $\left(N^{+}\right.$being the number of spins up and $N^{-}$the number of spins down). It is worth mentioning that $M$ is generally temperature dependent [89].

\subsection{Nonlinear response: Phase-space methods}

In order to investigate the nonlinear regime of the charge and spin dynamics, a phase-space approach is particularly interesting. In this paragraph, we will construct a Wigner equation that includes spin effects in the local density approximation, and show that its classical limit takes the form of a Vlasov equation.

The starting point for the derivation are the time-dependent Kohn-Sham (KS) equations described in Sec. 3.1. In terms of the Pauli 2-spinors

$$
\Psi_{i}(\mathbf{r}, \mathbf{t})=\left(\begin{array}{c}
\Psi_{i}^{\uparrow}(\mathbf{r}, \mathbf{t}) \\
\Psi_{i}^{\downarrow}(\mathbf{r}, \mathbf{t})
\end{array}\right)
$$

the KS equations can be written as:

$$
i \hbar \frac{\partial \Psi_{i}}{\partial t}=\left[\left(-\frac{\hbar^{2}}{2 m} \nabla^{2}+V(\mathbf{r}, t)\right) \mathbf{I}+\mu_{B} \boldsymbol{\sigma} \cdot \boldsymbol{B}(\mathbf{r}, t)\right] \Psi_{i}(\mathbf{r}, t)
$$

where $V(\mathbf{r}, t)=V_{\text {ext }}(\mathbf{r}, t)+V_{H}(\mathbf{r}, t)+V_{\mathrm{xc}}^{0}(\mathbf{r}, t), \mu_{B}$ is Bohr's magneton, $\boldsymbol{\sigma}=$ $\left(\sigma_{x}, \sigma_{y}, \sigma_{z}\right)$ are the $2 \times 2$ Pauli matrices, and $\mathbf{I}$ is the identity matrix. Here, $V_{\text {ext }}$ is an external potential (e.g. ionic jellium, external electric field, ...), $V_{H}$ is the Hartree potential that obeys Poisson's equation, and $V_{\mathrm{xc}}^{0}$ is the scalar part of the exchange-correlation potential. The magnetic field $\boldsymbol{B}=\boldsymbol{B}_{\mathrm{ext}}+\boldsymbol{B}_{\mathrm{xc}}$ is composed of an external part and an 'internal' part that stems from the exchange and correlation energy [see Eq. (66)]. In the so-called 'collinear' approximation, the latter reduces to $\boldsymbol{B}_{\mathrm{xc}}=B_{\mathrm{xc}} \hat{z}$. 


\section{Equation of motion for the density matrix}

By defining the density matrix

$$
\rho^{\eta \eta^{\prime}}\left(\mathbf{r}, \mathbf{r}^{\prime}\right)=\sum_{i} \Psi_{i}^{\eta}(\mathbf{r}) \Psi_{i}^{\eta^{\prime} *}\left(\mathbf{r}^{\prime}\right)
$$

where $\eta=\uparrow, \downarrow$, the KS equations (76) can be written in the following compact form (Von Neumann equation):

$$
i \hbar \frac{\partial \rho}{\partial t}=[H, \rho]
$$

where

$$
\rho=\left(\begin{array}{cc}
\rho^{\uparrow \uparrow} & \rho^{\uparrow \downarrow} \\
\rho^{\downarrow \uparrow} & \rho^{\downarrow \downarrow}
\end{array}\right) ; \quad H=\left(\begin{array}{cc}
h^{\uparrow \uparrow} & h^{\uparrow \downarrow} \\
h^{\downarrow \uparrow} & h^{\downarrow \downarrow}
\end{array}\right) .
$$

The only nondiagonal terms in the Hamiltonian come from the external or internal magnetic field $\boldsymbol{B}$.

We now introduce the following basis transformation for the Hamiltonian:

$$
H=h_{0} \mathbf{I}+\boldsymbol{h} \cdot \boldsymbol{\sigma}
$$

where $\boldsymbol{h}=\left(h_{x}, h_{y}, h_{z}\right)$, and

$$
\begin{aligned}
& h_{0}=\frac{h^{\uparrow \uparrow}+h^{\downarrow \downarrow}}{2}, h_{x}=\frac{h^{\uparrow \downarrow}+h^{\downarrow \uparrow}}{2} \\
& h_{z}=\frac{h^{\uparrow \uparrow}-h^{\downarrow \downarrow}}{2}, h_{y}=\frac{h^{\downarrow \uparrow}-h^{\uparrow \downarrow}}{2 i}
\end{aligned}
$$

For the Hamiltonian of Eq. (76), we have

$$
\begin{aligned}
& h_{0}(r)=-\frac{\hbar^{2}}{2 m} \nabla^{2}+V(r, t) \\
& h_{\alpha}(r)=\mu_{B} B_{\alpha}(r, t), \quad \alpha=x, y, z
\end{aligned}
$$

The same transformation (with identical notation) is also applied to the density matrix. With these definitions, the equations of motion for $\rho_{0}$ and $\rho_{\alpha}$ read as

$$
\begin{aligned}
i \hbar \partial_{t} \rho_{0} & =\left[h_{0}, \rho_{0}\right]+\sum_{\alpha=x, y, z}\left[h_{\alpha}, \rho_{\alpha}\right] \\
i \hbar \partial_{t} \rho_{\alpha} & =\left[h_{0}, \rho_{\alpha}\right]+\left[h_{\alpha}, \rho_{0}\right] .
\end{aligned}
$$

\section{'Spin' Wigner and Vlasov equations}

By making use of the Wigner transformation 


$$
\begin{aligned}
& f_{0}(\mathbf{r}, \mathbf{v}, t)=\frac{m}{2 \pi \hbar} \int d \lambda \rho_{0}\left(\mathbf{r}-\frac{\lambda}{2}, \mathbf{r}+\frac{\lambda}{2}\right) e^{i m \mathbf{v} \lambda / \hbar} \\
& f_{\alpha}(\mathbf{r}, \mathbf{v}, t)=\frac{m}{2 \pi \hbar} \int d \lambda \rho_{\alpha}\left(\mathbf{r}-\frac{\lambda}{2}, \mathbf{r}+\frac{\lambda}{2}\right) e^{i m \mathbf{v} \lambda / \hbar}
\end{aligned}
$$

one can easily obtain the equations of motion for the Wigner functions:

$$
\begin{aligned}
& \frac{\partial}{\partial t} f_{0}+\mathbf{v} \frac{\partial}{\partial \mathbf{r}} f_{0}- \\
& \frac{m}{2 i \pi \hbar^{2}} \int d \lambda \int d \mathbf{v}^{\prime} e^{i m\left(\mathbf{v}-\mathbf{v}^{\prime}\right) \lambda / \hbar}\left[V\left(\mathbf{r}+\frac{\lambda}{2}\right)-V\left(\mathbf{r}-\frac{\lambda}{2}\right)\right] f_{0}\left(\mathbf{r}, \mathbf{v}^{\prime}, t\right)- \\
& \sum_{\alpha} \frac{m \mu_{B}}{2 i \pi \hbar^{2}} \int d \lambda \int d \mathbf{v}^{\prime} e^{i m\left(\mathbf{v}-\mathbf{v}^{\prime}\right) \lambda / \hbar}\left[B_{\alpha}\left(\mathbf{r}+\frac{\lambda}{2}\right)-B_{\alpha}\left(\mathbf{r}-\frac{\lambda}{2}\right)\right] f_{\alpha}\left(\mathbf{r}, \mathbf{v}^{\prime}, t\right)=0 \\
& \frac{\partial}{\partial t} f_{\alpha}+\mathbf{v} \frac{\partial}{\partial \mathbf{r}} f_{\alpha}- \\
& \frac{m}{2 i \pi \hbar^{2}} \int d \lambda \int d \mathbf{v}^{\prime} e^{i m\left(\mathbf{v}-\mathbf{v}^{\prime}\right) \lambda / \hbar}\left[V\left(\mathbf{r}+\frac{\lambda}{2}\right)-V\left(\mathbf{r}-\frac{\lambda}{2}\right)\right] f_{\alpha}\left(\mathbf{r}, \mathbf{v}^{\prime}, t\right)- \\
& \frac{m \mu_{B}}{2 i \pi \hbar^{2}} \int d \lambda \int d \mathbf{v}^{\prime} e^{i m\left(\mathbf{v}-\mathbf{v}^{\prime}\right) \lambda / \hbar}\left[B_{\alpha}\left(\mathbf{r}+\frac{\lambda}{2}\right)-B_{\alpha}\left(\mathbf{r}-\frac{\lambda}{2}\right)\right] f_{0}\left(\mathbf{r}, \mathbf{v}^{\prime}, t\right)=0
\end{aligned}
$$

The corresponding Vlasov equations are obtained in the classical limit $\hbar \rightarrow 0$ :

$$
\begin{array}{r}
\frac{\partial}{\partial t} f_{0}+\mathbf{v} \frac{\partial}{\partial \mathbf{r}} f_{0}-\frac{1}{m} \frac{\partial V}{\partial \mathbf{r}} \frac{\partial f_{0}}{\partial \mathbf{v}}-\frac{\mu_{B}}{m} \sum_{\alpha} \frac{\partial B_{\alpha}}{\partial \mathbf{r}} \frac{\partial f_{\alpha}}{\partial \mathbf{v}}=0 \\
\frac{\partial}{\partial t} f_{\alpha}+\mathbf{v} \frac{\partial}{\partial \mathbf{r}} f_{\alpha}-\frac{1}{m} \frac{\partial V}{\partial \mathbf{r}} \frac{\partial f_{\alpha}}{\partial \mathbf{v}}-\frac{\mu_{B}}{m} \frac{\partial B_{\alpha}}{\partial \mathbf{r}} \frac{\partial f_{0}}{\partial \mathbf{v}}=0
\end{array}
$$

with $\alpha=x, y, z$.

Within the collinear approximation, the equations for $\alpha=x, y$ vanish. In this case, it is more convenient revert to the original representation and use

$$
\begin{aligned}
& f_{\uparrow}=f_{0}+f_{z} \\
& f_{\downarrow}=f_{0}-f_{z} .
\end{aligned}
$$

The corresponding Vlasov equations then become

$$
\begin{aligned}
& \frac{\partial}{\partial t} f_{\uparrow}+\mathbf{v} \frac{\partial}{\partial \mathbf{r}} f_{\uparrow}-\frac{1}{m}\left(\frac{\partial V}{\partial \mathbf{r}}+\mu_{B} \frac{\partial B_{z}}{\partial \mathbf{r}}\right) \frac{\partial f_{\uparrow}}{\partial \mathbf{v}}=0 \\
& \frac{\partial}{\partial t} f_{\downarrow}+\mathbf{v} \frac{\partial}{\partial \mathbf{r}} f_{\downarrow}-\frac{1}{m}\left(\frac{\partial V}{\partial \mathbf{r}}-\mu_{B} \frac{\partial B_{z}}{\partial \mathbf{r}}\right) \frac{\partial f_{\downarrow}}{\partial \mathbf{v}}=0 .
\end{aligned}
$$

The above Wigner and Vlasov equations can be used to study the nonlinear spin dynamics in a ferromagnetic nanoparticle or thin film, using numerical techniques similar to those employed for the electron dynamics. In their 
present form, these equations preserve the total spin, and thus cannot be used to describe the loss of magnetization observed in experiments [24]. A proper generalization, along the lines of the e-e and e-ph collision operators detailed in Sec. 2.3, would be necessary to account for these effects.

\section{Numerical example: the nonlinear many-electron dynamics in an anharmonic quantum well}

In order to illustrate qualitatively the practical implementation of the models described in the previous sections, we concentrate on a specific - and relatively simple - example. We consider an electron population confined in a one-dimensional anharmonic well defined by the potential

$$
V_{\text {conf }}(x)=\frac{1}{2} \omega_{0}^{2} m_{*} x^{2}+\frac{1}{2} K x^{4},
$$

where $m_{*}$ is the effective electron mass. The frequency $\omega_{0}$ can be related to a fictitious homogeneous positive charge of density $n_{0}$ via the relation $\omega_{0}^{2}=e^{2} n_{0} / m_{*} \varepsilon$. The total potential seen by the electrons is the sum of the confining potential $V_{\text {conf }}$ and the Hartree potential, which obeys Poisson's equation

$$
\frac{\partial^{2} V_{H}}{\partial x^{2}}=\frac{e^{2}}{\varepsilon} \int_{-\infty}^{\infty} f d v,
$$

where $e$ is the absolute electron charge and $\varepsilon$ is the effective dielectric constant. As initial condition, we take a Maxwell-Boltzmann distribution with Gaussian density profile

$$
f_{0}(x, v)=\frac{\bar{n}_{e}}{\sqrt{2 \pi k_{B} T_{e} / m_{*}}} \exp \left(-\frac{m_{*} v^{2}+m_{*} \omega_{0}^{2} x^{2}}{2 k_{B} T_{e}}\right),
$$

with temperature $T_{e}$ and peak density $\bar{n}_{e}$.

The electron dynamics is mainly determined by two dimensionless parameters: (i) the 'filling fraction' $\eta=\bar{n}_{e} / n_{0}=\omega_{p}^{2} / \omega_{0}^{2}$, which is a measure of self-consistent effects (in the limit case $\eta=0$, corresponding to very dilute electron densities, the Hartree potential is negligible); and (ii) the normalized Planck constant $H=\hbar \omega_{0} / k_{B} T_{e}$, which determines the importance of quantum effects. Notice that a small value of $H$ corresponds to a large electron temperature.

We use typical parameters for semiconductor quantum wells [90, 91]: effective electron mass and dielectric constant $m_{*}=0.067 m_{e}$ and $\varepsilon=13 \varepsilon_{0}$; volume density $n_{0}=10^{16} \mathrm{~cm}^{-3}$, oscillator energy $\hbar \omega_{0}=3.98 \mathrm{meV}$, and oscillator length $L_{\mathrm{ho}}=\sqrt{\hbar / m_{*} \omega_{0}} \simeq 17 \mathrm{~nm}$. For $\eta=1$, this yields a maximum surface density for the electrons $n_{s}=4.64 \times 10^{10} \mathrm{~cm}^{-2}$ and a maximum Fermi temperature $T_{F}=29.3 \mathrm{~K}$. A low electron temperature $T_{e} \simeq 46 \mathrm{~K}$ then yields $H \simeq 1$, whereas at room temperature $T_{e} \simeq 300 \mathrm{~K}$ one has $H \simeq 0.15$. 
The electron dynamics is excited by shifting the electron density of a finite distance $\delta x=L_{\mathrm{ho}}$. We will primarily be interested in the relaxation of the electric dipole, defined as the center of mass of the electron population: $d(t)=\iint f x d x d v / \iint f d x d v$, and of the average kinetic energy $E_{\mathrm{kin}}=\frac{1}{2} \iint f m_{*} v^{2} d x d v / \iint f d x d v$.

First, we present results obtained from the numerical resolution of the Wigner equation (37), coupled to Poisson's equation (94). The results were obtained with a numerical code that combines the split-operator method with fast Fourier transforms in the velocity coordinate [92]. We explore the electron dynamics for different values of the two relevant dimensionless parameters, $H$ and $\eta$. The anharmonicity parameter appearing in the confining potential (93) is fixed to $K=0.1$ (in units where $\hbar=m_{*}=\omega_{0}=1$ ). If the confinement were purely harmonic (i.e., $K=0$ ), the dipole would simply oscillate at the frequency $\omega_{0}$ irrespective of the value of the filling fraction. This result goes under the name of Kohn's theorem [31], and we have checked that it holds for our numerical simulations. When the confinement is not harmonic, the dipole should decay because of phase mixing effects.

The numerical results are shown in Fig. 8 (dipole) and Fig. 9 (kinetic energy). The fast oscillations correspond to the center of mass of the electron gas oscillating in the anharmonic well. For low electron densities and large temperatures $\left(\eta=0.1, T_{e}=300 \mathrm{~K}\right)$, the dipole relaxes to the bottom of the well, $d \simeq 0$, whereas the kinetic energy relaxes to a constant asymptotic value. This is a semiclassical regime where the energy spectrum is almost continuous: the observed relaxation is due to phase mixing effects.

Decreasing the temperature $\left(T_{e}=46 \mathrm{~K}\right)$ while keeping the density low $(\eta=0.1)$ produces a revival that occurs after the kinetic energy has initially relaxed. This is a typically quantum effect resulting from the discrete nature of the energy spectrum. The revival is clearly visible on the kinetic energy, but not so much on the dipole. When the electron density is large $(\eta=1)$, selfconsistent electron-electron interactions (Hartree potential) prevent the dipole and the kinetic energy from relaxing completely, even at large temperatures.

Next, we have added a dissipative term to the Wigner equation, in order to model electron-phonon (e-ph) collisions. This model has been discussed in Sec. 2.3. The relaxation rate is chosen to be $\gamma=0.001 \omega_{0}$, yielding a realistic relaxation time $\tau_{1}=\gamma^{-1} \simeq 165$ ps. The velocity-space diffusion coefficient is $D_{v}=\gamma v_{t h}$, where the thermal velocity is $v_{t h}=\sqrt{k_{B} T_{e} / m_{*}}$. The relaxation time $\tau_{2}$ depends on the velocity scale: for instance, a velocity scale $\Delta v$ is damped on a time scale $\tau_{2}=\tau_{1} \Delta v / v_{t h}$. Therefore, for velocity scales smaller than the thermal velocity, the decoherence time is always smaller than the relaxation time, in accordance with experimental findings.

We simulated the low temperature scenario $\left(T_{e}=46 \mathrm{~K}\right)$ in the presence of e-ph collisions, and observed that the revival occurring in the kinetic energy for $\eta=0.1$ is now suppressed (see Fig. 10). For large densities, however, the coherence of the electron motion is not lost, and the relaxation of the dipole 

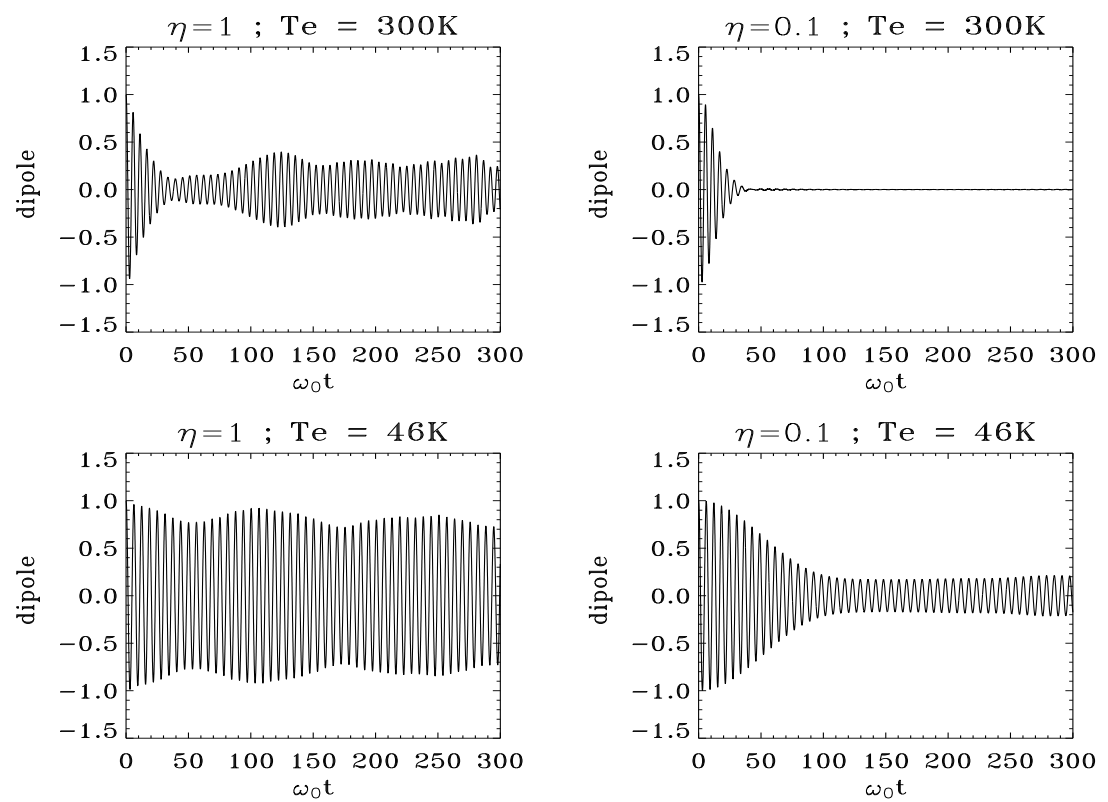

Fig. 8. Evolution of the electric dipole (in units of $L_{\mathrm{ho}}=17 \mathrm{~nm}$ ) obtained from the Wigner-Poisson model, for several values of $\eta$ and the electron temperature. Time is normalized to the oscillator frequency.

and the kinetic energy is only marginally faster compared to the collisionless regime.

Finally, we want to consider the zero-temperature case. For doing this, we resort to the hydrodynamical model described in Sec. 2.4. The relevant dimensionless parameters now are $\eta$ and $r_{s 0}$, the normalized Wigner-Seitz radius computed with the background density $n_{0}$. For $n_{0}=10^{16} \mathrm{~cm}^{-3}$, one has $r_{s 0}=2.8$. In Fig. 11 we plot the evolution of the electric dipole for different values of the filling fraction. Now, even for low electron densities, the dipole oscillates indefinitely without any appreciable decay. For larger electron densities, the motion is even more regular. It appears, therefore, that the dynamics becomes more and more regular as the electron temperature decreases, i.e. when quantum effect become more important. As mentioned above, this is essentially due to phase mixing effect, which become increasingly important in the semiclassical regime, where the energy levels are almost continuous.

\section{Conclusions and Perspectives}

In this review paper, we have presented some of the most common theoretical models used to describe the charge and spin dynamics in metallic and 

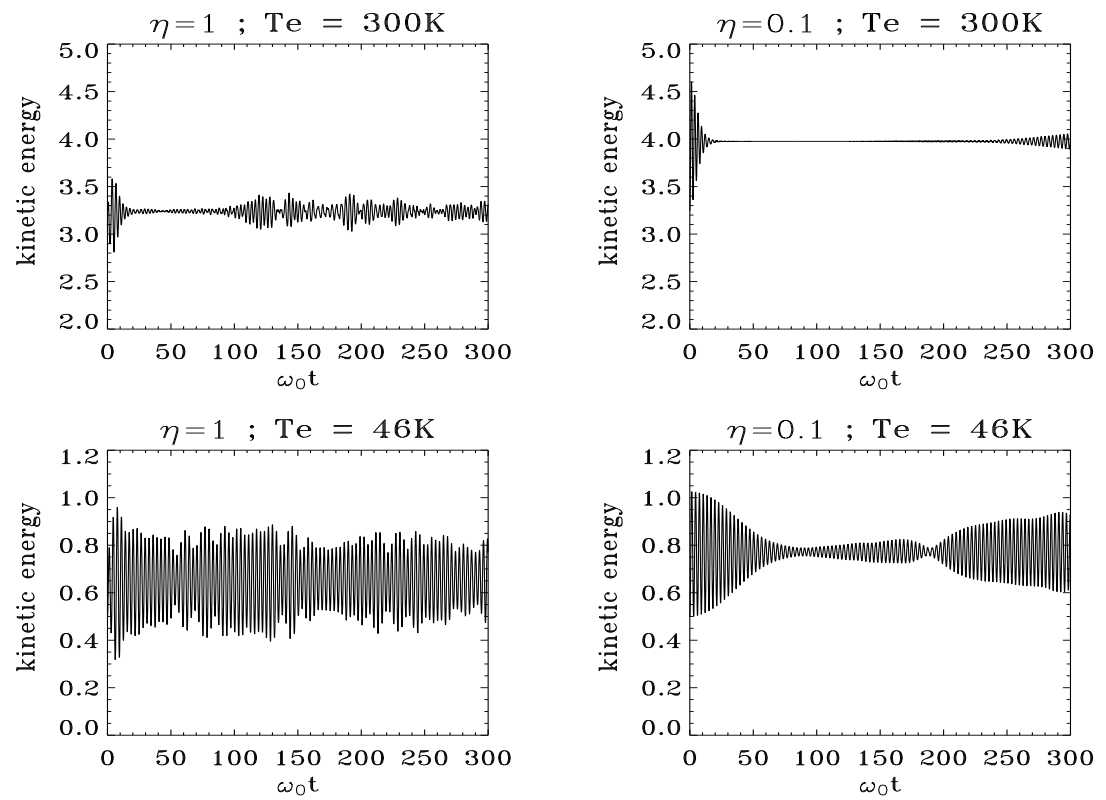

Fig. 9. Evolution of the kinetic energy (normalized to $\hbar \omega_{0}=3.98 \mathrm{meV}$ ) obtained from the Wigner-Poisson model, for several values of $\eta$ and the electron temperature. Time is normalized to the oscillator frequency.

semiconductor nanostructures. Three levels of description have been identified (see Fig. 1): (i) the full quantum $N$-body problem, which can only be addressed for small systems by using, for instance, the Configuration Interaction (CI) method; (ii) mean field models (Hartree and Wigner) and their generalizations to include exchange and correlations (Hartree-Fock, density functional theory); and (iii) quantum hydrodynamical models, which describe the electron dynamics via a small number of macroscopic variables, such as the density and the average velocity.

Each of these quantum-mechanical approaches has its classical counterpart: classical $N$-body models have been developed for molecular dynamics computations, as well as for gravitational $N$-body problems; classical mean field models are ubiquitous in plasma physics (Vlasov-Maxwell equations) and in the study of self-gravitating objects such as star clusters, galaxies, or even the entire universe; classical hydrodynamics hardly needs mentioning, as it is in itself an extremely wide field of research.

For each approach, we have stressed the difference between the linear and the nonlinear response. The former is valid for weak excitations and presupposes that the response is directly proportional to the excitation. Linear response theory is generally represented in the frequency domain. In contrast, nonlinear effects kick in for large excitations, and are best described in the time domain (this is because the time-frequency Fourier transform is a linear 

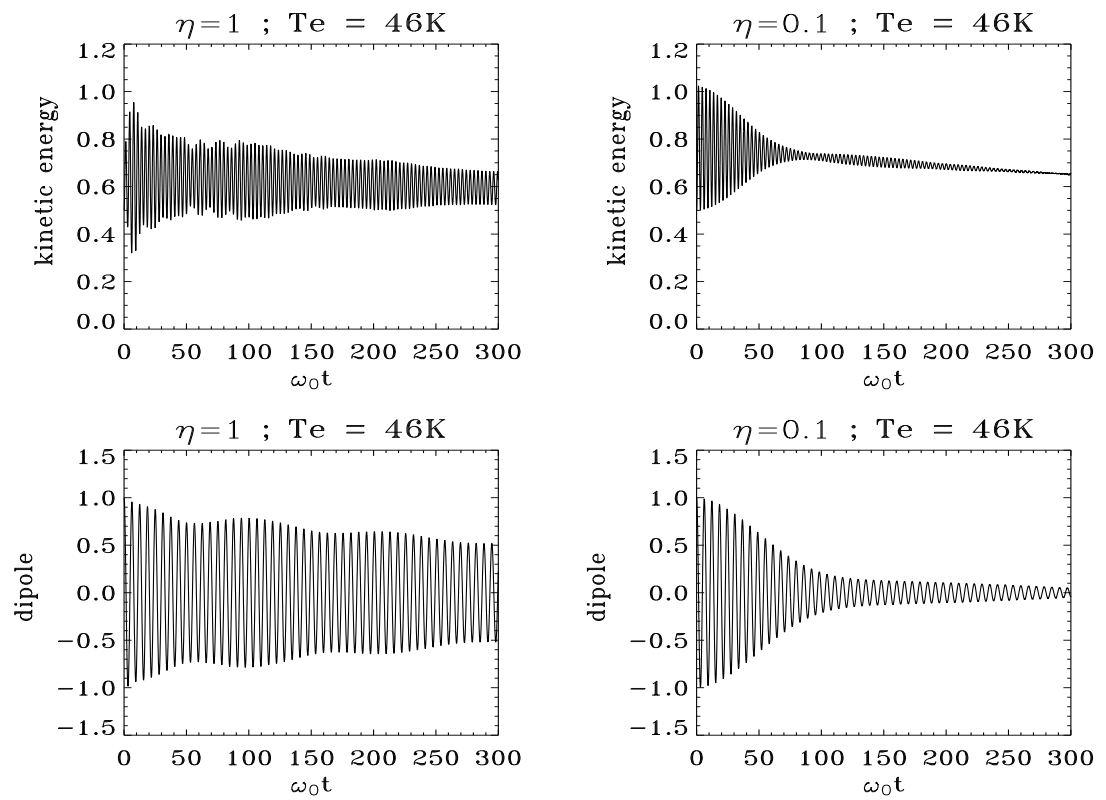

Fig. 10. Evolution of the kinetic energy (top panels) and electric dipole (bottom panels), from the Wigner-Poisson model including e-ph collisions. Same normalizations as in Figs. 8 and 9.
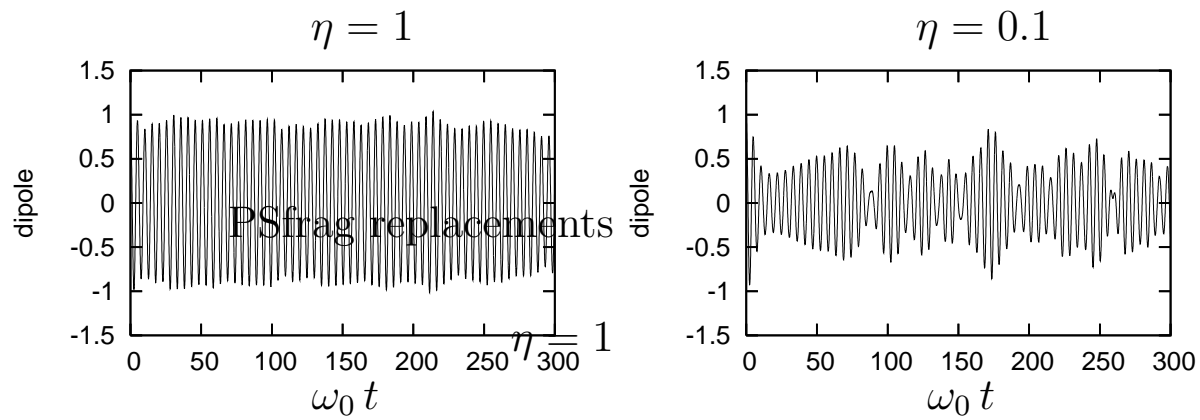

Fig. 11. Evolution of the electric dipole for $\eta=1$ (left frame) and $\eta=0.1$ (right frame), obtained from the quantum hydrodynamic model at $T_{e}=0$. 
operation, thus not adapted to describe nonlinear relations). Although a vast literature on the linear electronic response is available and dates back from the works of Drude in the early twentieth century, nonlinear effects have only been investigated in the last two decades, mainly with computer simulations.

The mean field level of description is perhaps the most widely used, as it incorporates, at least to lowest order, some of the features of the $N$-body dynamics, but still avoids the formidable complexity of the full problem. A particularly challenging open problem is the inclusion of dynamical correlations within mean-field models. Dynamical correlations differ from the correlations that are included in time-dependent density functional theory (TDDFT), inasmuch as they cannot be described by a slowly-varying density functional, as is done in ALDA (adiabatic local-density approximation). Whereas adiabatic correlations are described within an essentially Hamiltonian formulation and thus cannot model irreversible effects, dynamical correlations are responsible for the relaxation of the electron gas towards thermodynamical equilibrium. Some recent results have been obtained using a generalization of TDDFT that relies on the electron current as well as the electron density [42]. The phasespace approach, via the Wigner formulation, also appears promising to model effects beyond the mean field, as we have illustrated in Sec. 2.3.

Another important issue, which was not mentioned earlier in this review, is the inclusion of relativistic corrections in the above models for the electron dynamics. Spin-orbit coupling (which is an effect appearing at second order in $v / c)$ is sometimes taken into account in a semi-phenomenological way within the Pauli equation. However, other terms occurring at the same order are often neglected without further justification. A consistent derivation of relativistic effects to a certain order in $v / c$ can of course be carried out, starting from the Dirac equation, for the case of a single particle in an external electromagnetic field [93]. For a many-body system, this issue is much trickier and is the object of current investigations.

Nanostructures are by definition finite-size objects. Due to the presence of boundaries and interfaces, the electron dynamics can thus display novel and unexpected features compared to bulk matter. For example, as the elastic and inelastic scattering length $(\sim 10-50 \mathrm{~nm}$ for bulk metals) are much longer than the size of the system, an electron - or a group of electrons - can travel coherently through the length of the system, thus leading to ballistic transport between the surfaces. The theoretical tools to study finite-size nano-objects are also relatively recent, and have been developed alongside the experimental breakthroughs that made these objects widely available.

If the electron dynamics in nanosized objects has received considerable attention for the last thirty years, the spin dynamics is a much younger field of research, both experimentally and theoretically. Nevertheless, the already existing applications to memory storage and processing, and the still speculative, but highly enthralling, developments in quantum computing, have stimulated a large number of works in this direction. In Sec. 3 we have illustrated how the models for the electron dynamics can be extended to include the spin degrees 
of freedom, both in the linear and nonlinear regimes. An outstanding question concerns the demagnetization processes observed in ferromagnetic thin films irradiated with femtosecond laser pulses, for which a clear theoretical explanation is still lacking.

The field of optical control of spins in semiconductor nanostructures is also a very active research area. It is nowadays possible to fabricate and optically probe individual semiconductor quantum dots doped with one or more magnetic impurities [94]. One of the major interest of this type of structure is the possibility to control magnetism via optical processes acting on the charge carriers. Thus, ferromagnetism becomes optically manipulable on an ultrafast timescale. This is particulary interesting for the elaboration of future fastaccess magnetic storage devices. We are currently working on quasi one- and two-dimensional nonparabolic quantum dots containing up to four electrons and doped with a finite number of localized magnetic impurities. Within the framework of the CI method and the Anderson model, we aim at investigating the influence of the impurities on the energy spectra and oscillator strengths with special emphasis on the breakdown of the Kohn theorem.

Finally, another procedure that has attracted particular attention over the last decade is the low-density doping of semiconductor nanostructures with magnetic impurities such as manganese ions. The resulting materials (named DMS, for diluted magnetic semiconductors) can display Curie temperatures as high as $80 \mathrm{~K}$ [95], and possibly larger [96]. The spin of the Mn ions is coupled to the spin degrees of freedom of the electrons and holes, whose dynamics can be optically excited. DMS thus offer the possibility of using laser pulses to control the magnetization dynamics of semiconductor nanostructures.

Given the wealth of fundamental issues and practical applications, the interplay of charge and spin effects in nanosized objects is bound to remain a major area of research in the coming years.

\section{References}

1. G. L. Eesley, Phys. Rev. Lett. 51, 2140 (1983).

2. S. D. Brorson, J. G. Fujimoto, and E. P. Ippen, Phys. Rev. Lett. 59, 1962 (1987).

3. C. Suárez, W. E. Bron, and T. Juhasz, Phys. Rev. Lett. 75, 4536 (1995).

4. R. H. M. Groeneveld, R. Sprik, and A. Lagendijk, Phys. Rev. B 51, 11433 (1995).

5. C.-K. Sun, F. Vallée, L. H. Acioli, E. P. Ippen and J. G. Fujimoto, Phys. Rev. B 50, 15337 (1994).

6. J.-Y. Bigot, V. Halté, J.-C. Merle, and A. Daunois, Chem. Phys. 251, 181 (2000).

7. M. Bauer and M. Aeschlimann, J. Electr. Spectr. 124, 225 (2002).

8. W. Rudolph, P. Dorn, X. Liu, N. Vretenar and R. Stock, Applied Surface Science 208-209, 327 (2003).

9. J-S. Lauret, C. Voisin, G. Cassabois, C. Delalande, Ph. Roussignol, O. Jost, and L. Capes, Phys. Rev. Lett. 90, 057404 (2003). 
10. R. Schlipper, R. Kusche, B. v. Issendorff, H. Haberland, Appl. Phys. A 72, 255259 (2001).

11. E. E. B. Campbell, K. Hansen, K. Hoffmann, G. Korn, M. Tchaplyguine, M. Wittmann, and I. V. Hertel, Phys. Rev. Lett. 84, 2128 (2000).

12. C. Voisin, D. Christofilos, N. Del Fatti, F. Vallée, B. Prével, E. Cottancin, J. Lermé, M. Pellarin, and M. Broyer, Phys. Rev. Lett. 85, 2200 (2000).

13. M. Nisoli, S. Stagira, S. De Silvestri, A. Stella, P. Tognini, P. Cheyssac, and R. Kofman, Phys. Rev. Lett. 78, 3575 (1997).

14. B. Lamprecht, J. R. Krenn, A. Leitner, and F. R. Aussenegg, Phys. Rev. Lett. 83, 4421 (1999).

15. U. Kreibig and M. Vollmer, Optical Properties of Metal Clusters (Springer, New York, 1995).

16. R. A. Molina, D. Weinmann, and R. A. Jalabert, Phys. Rev. B 65, 155427 (2002).

17. D. F. Zaretsky, Ph. A. Korneev, S. V. Popruzhenko, and W. Becker, J. Phys. B 37, 4817 (2004).

18. G. Manfredi and P.-A. Hervieux, Phys. Rev. B 70, 201402(R) (2004); Phys. Rev. B 72, 155421 (2005); Optics Lett. 30, 3090 (2005).

19. J. F. Dobson, Phys. Rev. B 46, 10163 (1992).

20. D. Loss, D. P. DiVincenzo, Phys. Rev. A 57, 120 (1998).

21. P. Zoller et al., Eur. J. Phys. 36, 203 (2005).

22. J. Gorman, D. G. Hasko, and D. A. Williams, Phys. Rev. Lett. 95, 090502 (2005).

23. J. R. Petta, A. C. Johnson, C. M. Marcus, M. P. Hanson, and A. C. Gossard, Phys. Rev. Lett. 93, 186802 (2004).

24. E. Beaurepaire, J. -C. Merle, A. Daunois, J.-Y. Bigot, Phys. Rev. Lett. 76, 4250 (1996); J.-Y. Bigot, L. Guidoni, E. Beaurepaire, and P. N. Saeta, Phys. Rev. Lett. 93, 077401 (2004); L. Guidoni, E. Beaurepaire, and J.-Y. Bigot, Phys. Rev. Lett. 89, 017401 (2002).

25. G. Zhang and W. Hübner, Phys. Rev. Lett. 85, 3025 (2000).

26. B. Koopmans, J. J. M. Ruigrok et al., Phys. Rev. Lett. 95, 267207 (2005).

27. R. Balescu, Equilibrium and Nonequilibrium Statistical Mechanics (Wiley, New York, 1975).

28. P. Hohenberg and W. Kohn, Phys. Rev. B 136, B864 (1964); W. Kohn and L. J. Sham, Phys. Rev. 140, A1133 (1965).

29. E. P. Wigner, Phys. Rev. 40, 749 (1932).

30. C. D. Sherrill and H. F. Schaefer, Adv. Quantum Chem. 34, 143 (1999).

31. W. Kohn, Phys. Rev. 123, 1242 (1961).

32. T. Sako, P.-A. Hervieux, and G. H. F. Diercksen, Phys. Rev. B 74, 045329 (2006).

33. T. Sako and G. H. F. Diercksen, J. Phys. B: At. Mol. Opt. Phys. 36, 1433 (2003); J. Phys. B: At. Mol. Opt. Phys. 36, 1681 (2003).

34. E. Runge and E. K. U. Gross, Phys. Rev. Lett. 52, 997 (1984).

35. M. E. Casida, In: Recent Developments and Applications of Modern Density Functional Theory vol 4, ed by J. M. Seminario (Elsevier, Amsterdam, 1996)

36. E. K. U. Gross, J. F. Dobson, and M. Petersilka, In: Topics in Current Chemistry (Springer, Berlin, 1996), pp 81-172.

37. S. Lundqvist and N. H. March: Theory of the inhomogeneous electron gas (Plenum Press, New York, 1983). 
38. G. Onida, L. Reining, and A. Rubio, Rev. Mod. Phys. 74, 601 (2002).

39. J. F. Dobson, Phys. Rev. Lett. 73, 2244 (1994).

40. E. K. U. Gross and W. Kohn, Adv. Quant. Chem. 21, 255 (1990).

41. G. Vignale and W. Kohn, Phys. Rev. Lett. 77, 2037 (1996).

42. R. D’Agosta and G. Vignale, Phys. Rev. Lett. 96, 016405 (2006).

43. G. Vignale and W. Kohn: Electronic Density Functional Theory, ed by J. Dobson, M. P. Das, and G. Vignale (Plenum Press, New York, 1997).

44. G. Vignale and M. Rasolt, Phys. Rev. Lett. 59, 2360 (1987).

45. F. Calvayrac, P.-G. Reinhard, E. Suraud, and C. Ullrich, Phys. Rep. 337, 493 (2000).

46. B. Gervais, E. Giglio, A. Ipatov, J. Douady, Comp. Mat. Sc. 35, 359 (2006).

47. L. F. Ruiz, P. -A. Hervieux, J. Hanssen, M. F. Politis, and F. Martin, Int. J. Quantum Chem. 86, 106 (2002).

48. D. Bauer, F. Ceccherini, A. Macchi, and F. Cornolti, Phys. Rev. A 64, 063203 (2001).

49. E. Cormier, P.-A. Hervieux, R. Wiehle, B. Witzel and H. Helm, Eur. Phys. J. D 26, 83 (2003).

50. A. Zangwill and P. Soven, Phys. Rev. A 21, 1561 (1980).

51. Z. Levine and P. Soven, Phys. Rev. Lett. 50, 2074 (1983).

52. W. Eckardt, Phys. Rev. B 31, 6360 (1985).

53. J. Lermé et al., Eur. Phys. J. D 4, 95 (1998).

54. A. G. Eguiluz, Phys. Rev. Lett. 51, 1907 (1983).

55. E. Lipparini and Ll. Serra, Phys. Rev. B 57, R6830 (1998).

56. N. D. Mermin, Phys. Rev. 137, A1441 (1965); W. Kohn and L. J. Sham, Phys. Rev. 140, A1133 (1965); U. Gupta and A. K. Rajagopal, Phys. Rep. 87, 259 (1982); W. Yang, Phys. Rev. A 38, 5504 (1988).

57. L. D. Landau and E. M. Lifchitz, Statistical physics (Pergamon Press, Oxford, 1969).

58. P.-A. Hervieux, A. Benabbas, V. Halté, and J. -Y. Bigot, Eur. Phys. J. D 24, $185(2003)$.

59. P.-A. Hervieux and J. -Y. Bigot, Phys. Rev. Lett. 92, 197402 (2004).

60. M. Petersilka et al., Phys. Rev. Lett. 76, 1212 (1996).

61. W. Yang, Phys. Rev. A 38, 5512 (1988).

62. G. D. Mahan and K. R. Subbaswamy, Local Density Theory of Polarizability (Plenum Press, New York, 1990).

63. J. G. Fujimoto et al., Phys. Rev. Lett. 53, 1837 (1984); L. Jiang and H. -L. Tsai, J. Heat Transfer 127, 1167 (2005).

64. F. Vallée, J. Phys. Chem. B 105, 2264 (2001).

65. N. W. Ashcroft and N. D. Mermin, Solid state physics (Saunders College Publishing, Orlando, 1976).

66. Thanh N. Truong et al: J. Chem. Phys. 96, 2077 (1992).

67. J. E. Moyal, Proc. Cambridge Phil. Soc. 45, 99 (1949).

68. V. I. Tatarskii, Sov. Phys. Usp. 26, 311 (1983) [Usp. Fis. Nauk. 139, 587 (1983)].

69. M. Hillery, R. F. O'Connell, M. O. Scully, and E. P. Wigner, Phys. Rep. 106, 121 (1984).

70. G. Manfredi and M. R. Feix, Phys. Rev. E 53, 6460 (1996).

71. N. C. Kluksdahl, A. M. Kriman, D. K. Ferry, and C. Ringhofer, Phys. Rev. B 39, 7720 (1989).

72. P. A. Markowich, C. A. Ringhofer, and C. Schmeiser, Semiconductor equations (Springer, Vienna, 1990). 
73. J. E. Drummond, Plasma Physics (McGraw- Hill, New York, 1961).

74. J. Lindhard, K. Dan. Vidensk. Selsk. Mat. Fys. Medd. 28, 1 (1954).

75. X. Liu, R. Stock, and W. Rudolph, Phys. Rev. B 72, 195431 (2005).

76. M. Anderegg, Phys. Rev. Lett. 27, 1575 (1971).

77. R. Jasiak, G. Manfredi, and P.-A. Hervieux, in preparation.

78. W. S. Fann, R. Storz, H. W. K. Tom, and J. Bokor, Phys. Rev. B 46, 13592 (1992).

79. E. A. Uehling et al., Phys. Rev. 43, 552 (1933)

80. A. Domps, P.-G. Reinhard and E. Suraud Phys. Rev. Lett. 81, 5524 (1998).

81. G. Lindblad, Commun. Math. Phys. 48, 119 (1976).

82. D. Pines and P. Nozières, The theory of quantum liquids (W. A. Benjamin, New York, 1966).

83. W. H. Zurek, Rev. Mod. Phys. 75, 715 (2003).

84. A. Isar, A. Sandulescu, H. Scutaru, E. Stefanescu and W. Scheid, Int. J. Mod. Phys. E 3, 635 (1994).

85. G. Manfredi and F. Haas, Phys. Rev. B 64, 075316 (2001).

86. N. Crouseilles, P.-A. Hervieux, and G. Manfredi, in preparation.

87. A. K. Rajagopal, Phys. Rev. B 17, 2980 (1978).

88. M. I. Katsnelson and A. I. Lichtenstein, J. Phys.: Condens. Matter 16, 7439 (2004).

89. E. Maurat and P.-A. Hervieux, submitted to Phys. Rev. B.

90. H. O. Wijewardane and C. A. Ullrich, Appl. Phys. Lett. 84, 3984 (2004).

91. G. Manfredi and P.-A. Hervieux, Appl. Phys. Lett. 91, 061108 (2007).

92. N. Suh, M. R. Feix, and P. Bertrand, J. Comput. Phys. 94 (1991), 403.

93. L. D. Landau and E. M. Lifschitz, Quantum Electrodynamics (Pergamon Press, Oxford, 1983); P. Strange, Relativistic Quantum Mechanics (Cambridge University Press, Cambridge, 1998).

94. L. Besombes et al, Phys. Rev. Lett 93, 207403 (2004); Y. Leger et al., Phys. Rev. Lett. 97, 107401 (2006).

95. J. Wang et al., Phys. Rev. Lett. 98, 217401 (2007).

96. T. Dietl et al., Science 287, 1019 (2000). 